Linköping Studies in Science and Technology Thesis No. 1738

\title{
Charge Transport Simulations for Organic Electronics: A Kinetic Monte Carlo Approach
}

\author{
Riccardo Volpi
}

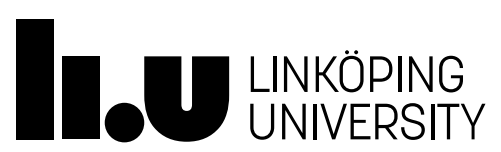

Department of Physics, Chemistry, and Biology (IFM)

Linköping University, SE-581 83 Linköping, Sweden

Linköping 2016 
ISBN 978-91-7685-878-3

ISSN 0280-7971

Printed by LiU-Tryck 2016 


\section{Abstract}

In this thesis we focus on the modelling and simulation of organic electronic devices, investigating their structural and electronic properties. Organic devices have attracted great interest for their innovative properties, but their functioning still represent a theoretical and technological challenge. They are composed by one or more organic materials depending on the particular application. The morphology of organic devices in the single phase or at the interface is known to strongly determine mobility and efficiency of the devices. The structural disorder is studied through molecular dynamics (MD) simulations. Marcus formula is used to calculate the hopping rate of the charge carriers and the model developed is tested by simulations in a Kinetic Monte Carlo scheme. The dependence of the transfer integrals on the relative molecular orientation is achieved through a weighted Mulliken formula or through a dimer projection approach using the semi-empirical Hartree Fock method ZINDO. Electrostatic effects, have been included through atomic charges and atomic polarizabilities, calculated at the B3LYP level of theory. The inclusion of electrostatic effects has been shown (through simulations in $4 \mathrm{PV}$ and $\mathrm{C}_{60}$ ) to be crucial to obtain a good qualitative agreement with experiments, for both mobility field and temperature dependence in the single phase. In particular the external reorganization energy, calculated through the polarization of the environment, has been shown to have a great impact on the conduction, shifting the inverse Marcus region and helping $\mathrm{CT}$ state separation at the interface (between $\mathrm{C}_{60}$ and anthracene). 


\section{Acknowledgements}

I would like to thank my supervisor Mathieu Linares for helping me since the beginning in getting started with this new adventure here in Sweden. Thanks for being always a great source of ideas, open to discussion and pushing me to do better. Thanks to my co-supervisor Sven Stafström, for being always so insightful during the conversations we had.

I would like to thank past and present colleagues of the Theoretical Chemistry (former Computational Physics) group, for the awesome fikas and entertaining conversations. Thanks to Patrick Norman for promoting such a scientifically interesting and at the same time relaxed environment. Thanks to Bo Durbeej for the stimulating teaching opportunity he has given me. Also, thanks to the administration at IFM and in particular to Lejla Kronbäck for the great work.

Thanks to the many friends, met along the way, for making these years more light and fun (sorry if i cannot mention everybody). In particular thanks (in order of appearance): to Rafael for the great eurotrips and the precision in communicating the time; to Raul for the funny challenges and the interesting fika case studies; and to Hassan for the great costumes and for sharing russian randomness with me.

Finally, a great thanks to my family, for the constant love and support, that is in no way altered by the distance. 

1 Introduction 1

1.1 Organic Molecules and Polymers . . . . . . . . . . . . 2

1.2 Organic Devices . . . . . . . . . . . . . . . . . . . . . . . . . . . . . . . . .

1.3 Modelling Challenges . . . . . . . . . . . . . . . . 7

2 A Realistic Molecular Arrangement 9

2.1 Force field energetic contributions . . . . . . . . . . . . . . . . . 10

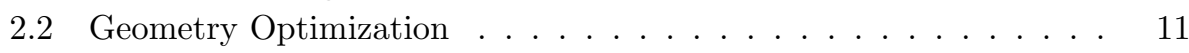

2.3 Molecular Dynamics . . . . . . . . . . . . . . . . 11

2.4 Canonical Ensembles . . . . . . . . . . . . . . . . . . 13

2.4.1 Berendsen temperature coupling . . . . . . . . . 13

2.4.2 Nosé-Hoover temperature coupling . . . . . . . . . . . . 14

2.4.3 Berendsen pressure coupling . . . . . . . . . . 15

2.4.4 Parrinello-Rahman pressure coupling . . . . . . . . . 16

2.5 The Ising Model . . . . . . . . . . . . . . . . . . . . . . . . . . 17

3 Molecular Orbitals Theory $\quad 19$

3.1 Born-Oppenheimer Approximation . . . . . . . . . . . . . 19

3.2 Symmetrization and Antisymmetrization Operators . . . . . . . . 21

3.3 Hartree-Fock . . . . . . . . . . . . . . . . . . . . . . 22

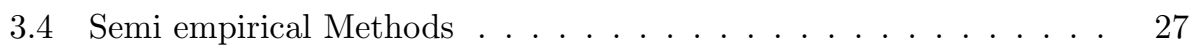

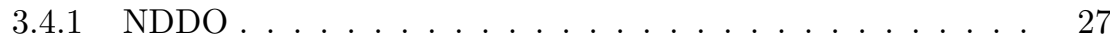

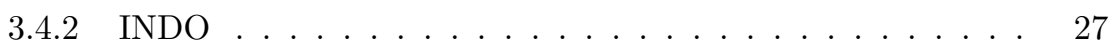

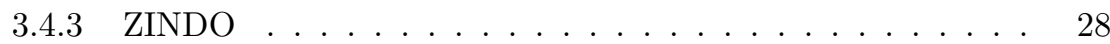

3.5 Density Functional Theory . . . . . . . . . . . . . 28

3.5.1 Kohn-Sham Scheme . . . . . . . . . . . . . . 30 
4 Charge Transport in Organic Materials 33

4.1 Franck-Condon Principle . . . . . . . . . . . . . . . . 34

4.2 Vibrational coupling on a single molecule . . . . . . . . . . . 35

4.3 Holstein and Holstein-Peierls Hamiltonian . . . . . . . . . . . . . . 38

4.4 Marcus Formula . . . . . . . . . . . . . . . 40

5 Monte Carlo Simulations $\quad 43$

5.1 Kinetic Monte Carlo Algorithm . . . . . . . . . . . . . . . . . . 44

5.2 Marcus Parameters Calculation . . . . . . . . . . . . . 46

5.2 .1 Site Energies . . . . . . . . . . . . . . . . 46

5.2 .2 Transfer Integrals . . . . . . . . . . . . . . . . . . . . . 48

5.2.3 Reorganization Energies . . . . . . . . . . . . 49

6 Summary and Results $\mathbf{5 1}$

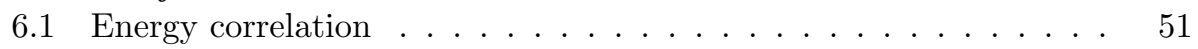

6.2 Polarization . . . . . . . . . . . . . . . . . 52

6.3 CT state splitting . . . . . . . . . . . . . . . . . 52

Bibliography $\quad 55$

$\begin{array}{ll}\text { List of included Publications } & 63\end{array}$

$\begin{array}{ll}\text { Paper I } & 65\end{array}$

$\begin{array}{ll}\text { Paper II } & 79\end{array}$

$\begin{array}{ll}\text { Paper III } & 111\end{array}$

A The Virial Theorem 135 


\section{CHAPTER 1}

\section{Introduction}

Electronics is the branch of physics dealing with the design and the interconnection of electrical components. With the advancement of technology, electronic devices are progressively acquiring importance in our everyday life, with key components being transistors, LEDs and solar cells. Electronic devices contain an active semiconductor that is traditionally silicon or another inorganic material (Ge, GaAs, etc.). However, silicon is far from being an eco-friendly material [1] and a mass production of devices based on this material cannot be a long-term solution. Silicon production (in its pure and crystalline form) requires high amount of energy and environmentally harmful chemicals. Furthermore improper disposal of silicon devices at the end of their lifetime presents an environmental and health concern. In looking for alternative materials, nature represents a good source of inspiration. Carbon is a light abundant element, and with its four valence electrons is at the base of the complexity of all life-forms. Photosynthesis, as an example, has been slowly refined through billions of years till arriving to the efficient light-harvesting process that we are witnessing today. Also, carbon is belonging to the group IV of the periodic table, just on top of silicon, and it can be expected to have similar chemical properties.

Organic Electronics is a branch of Electronics based on organic materials, compounds containing carbon and hydrogen. Organic semiconductors are light, flexible and transparent, allowing for the design of new futuristic devices [2-4]. Organic materials are also environmentally safe and low cost to produce since they can be obtained by spin coating, ink-jet printing, lithography, etc. without the need of high temperatures or vacuum. Organic light-emitting diodes (OLEDs) and organic field effect transistors (OFETs) are already in commercial production and employed for thin displays and other simple devices. The performance of these organic components, "in some cases has already approached or even exceeded the requirements of the particular application" [2]. More importantly, cheap and 
low-power-consumption circuitry allows the integration of disposable and recyclable electronics in our everyday life [5], opening the door for innovative and revolutionary technologies.

One of the most important challenges of organic electronics is represented by organic solar cells, or organic photovoltaics (OPV). This is a problem of great importance also from an energetic point of view, another hot topic in modern society. In the search for an abundant and clean energy source, the Sun represents the ideal candidate. The total energy irradiated on Earth (5\% UV, $43 \%$ visible, 52 $\%$ IR) exceeds the world's energy consumption by several thousands times [6]. The efficient conversion of such energy in a usable form represents the main technological challenge. This difficult task is attempted through solar cells, optoelectronic devices that transform sunlight into electricity. Conventional solar cells are built from inorganic materials, mainly based on silicon, and their efficiencies are approaching the theoretical Shockley-Queisser limit [7] for single junction solar cell [8-11]. Also, as a result of the high-energies required to obtain pure and crystalline silicon, the cost of inorganic solar cells is generally too high to allow their extensive and integrated use in daily life.

Organic solar cells have reached a reported record efficiency of $11 \%$ [11]. While this is still not enough to have a commercial interest, the competition with inorganic solar cells is not focused mainly on the efficiency, but more on the innovative applications originating from the particular properties of organic materials (flexibility, transparency, etc.). Another of the current downsides of organic solar cells is the instability of organic semiconductors, decreasing the performances of the devices and ultimately their lifetimes [12].

\subsection{Organic Molecules and Polymers}

With the term organic materials we commonly refer to chemical compounds composed mainly by carbon and hydrogen. The semiconductive properties of these materials are principally determined by the four valence electrons of the carbon. The electronic configuration of a carbon atom in the ground state is $1 s^{2} 2 s^{2} 2 p^{2}$. The four electrons in the outer shell can form hybridized orbitals. The hybridization depends on the number of $\sigma$ bonds the carbon is making. When making four $\sigma$ bonds, we have $s p^{3}$ hybridization, characterized by the formation of four $s p^{3}$ orbitals in tetrahedral configuration. In case of three $\sigma$ bonds, $s p^{2}$ hybridization is happening. Three $s p^{2}$ orbitals lie on a plane (oriented at a $\pi / 3$ angle) while the remaining $p$ orbital is orthogonal to such plane. Two $\sigma$ bonds lead to $s p$ hybridization, two linear $s p$ orbitals are formed pointing in opposite directions and the other two $p$ orbitals remain unaltered.

The semiconductive behaviour of conjugated polymers is mainly determined by the $p$ orbitals not involved in the $\sigma$ bondings $[13,14] . \sigma$ bonds are formed between the $s p^{2}$ orbitals of the carbon atoms composing the backbone of the polymer; from which the typical planar configuration of this class of materials. The remaining $p$ orbitals are orthogonal to the plane of the molecule and form the alternating $\pi$ bonds. The electronic clouds of two such $p$ orbitals mix in a linear combination, 
that depending on the sign can produce a bonding $(\pi)$ or anti-bonding $\left(\pi^{*}\right)$ wave function. These $\pi$-electrons result in spatially delocalized electrons. They belong to the whole $\pi$-segment and not to a specific carbon atom, and are responsible for the conductivity of the molecule. A $\pi$-segment can be extended over the entire organic polymer or just over a part of it, depending on the specific polymer and its particular conformation. In general, all organic molecules present some similar behaviour, given by the presence of carbon chains in parts of their structure.

In the molecular ground state, the electrons fill the lowest energies orbitals with a maximum of two electrons of opposite spins each. In this way, for example for two carbon atoms, the two electrons that were in $p$ orbitals fill the $\pi$ level with opposite spins, while the $\pi^{*}$ orbitals remain empty. The molecular ground state is the highest occupied molecular orbital (HOMO) $\pi$ and the lowest unoccupied molecular orbital (LUMO) $\pi^{*}$ represent the first virtual orbital (fig. 1.1). The
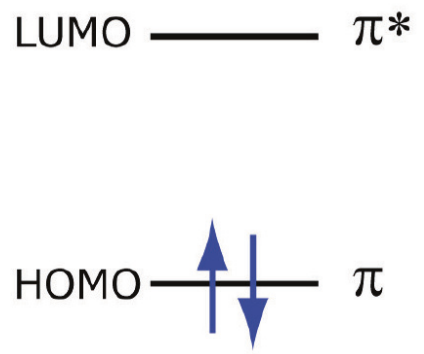

Figure 1.1. Highest occupied and lowest unoccupied molecular orbitals (HOMO and LUMO). Arrows denote two electrons with different spins.

ground state of the majority of organic molecules is electrically neutral and has total spin zero (is a singlet state). When one electron from the HOMO is raised to the LUMO, an exciton is formed on the molecule, usually localized on the $\pi$-segments of the molecule involved in the excitation. Electrons and holes are spin- $\frac{1}{2}$ particles, so the formed exciton can either be a singlet exciton or a triplet exciton.

\subsection{Organic Devices}

The charge transport in organic materials is different in respect to the inorganic ones for two main reasons. First, excited electrons are not free to move as in inorganic crystals. An excited electron and its respective hole remain bounded together in an hydrogenoid system commonly called exciton. This is due to the poor screening of charges in organic materials due to low dielectric constants (typically $\epsilon_{r} \simeq 3.5$ ). Second, organic materials are "soft". A single charge carrier, hopping in an organic material, strongly interact with the vibrations on its nearby molecules, creating quantum quasiparticles called polarons.

There is consensus on the main physical processes behind the functioning of organic devices, as has been described in several papers. A detailed discussion can 
be found in [15-17]. Here we will illustrate more in detail the processes happening in an organic solar cell (fig. 1.2). Many of these processes are common to all organic devices; in particular in an OLED exactly the same processes are happening, just in reversed order. (1) A photon is absorbed with probability $\eta_{a b s}$, generating an

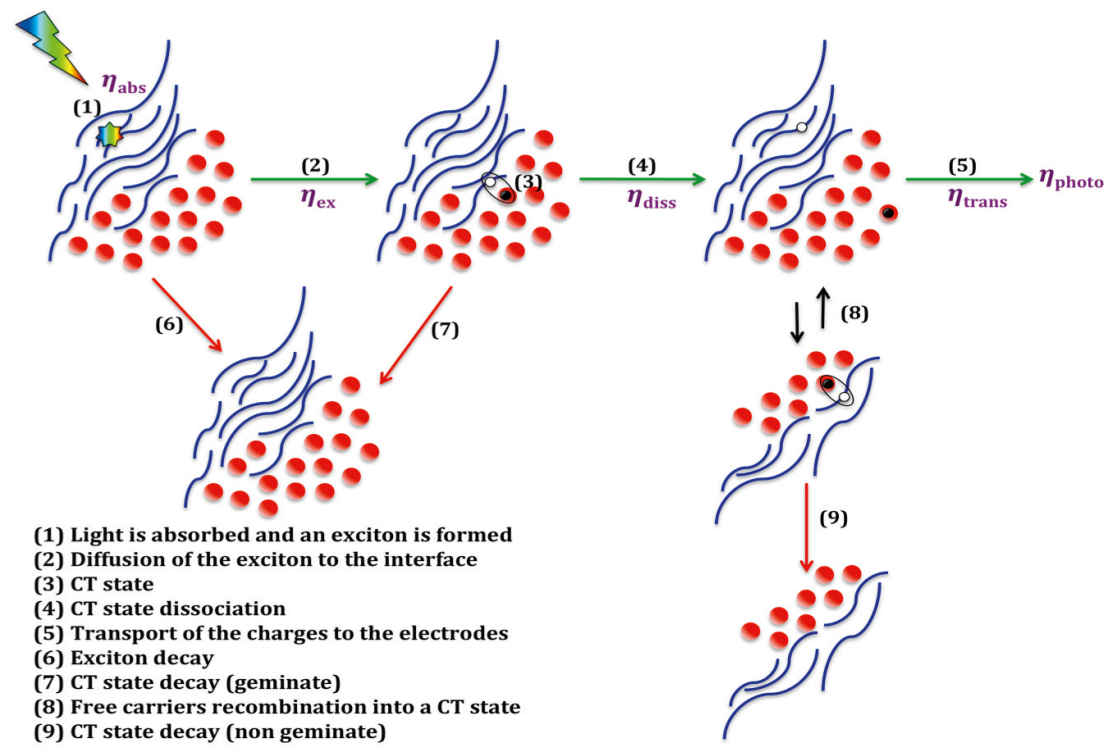

Figure 1.2. Schematic illustration of an organic solar cell working principles. Figure adapted from [16].

exciton. (2) The exciton diffuses to the interface with probability $\eta_{e x},(3)$ forming a charge transfer state (CT). (4) The CT state split into free electrons and holes with probability $\eta_{\text {diss }}$. (5) Now the charge carriers are free to move and can reach the electrodes with probability $\eta_{\text {trans }}$. Each one of these processes has some losses associated. (6) The exciton or (7 and 9) the CT state generated can recombine (radiatively) or (8) even free charge carriers can recombine in a CT state at the interface.

In the remaining part of this section we will present the main differences between organic and inorganic. After absorption of a photon, an exciton is created in the material, i.e. the hole and the electron formed after an excitation (fig. 1.3) form an hydrogenoid system, whose binding energy can be estimated as

$$
E_{e x}=\left(\frac{m}{m_{0}}\right) \frac{1}{\epsilon_{r}^{2}} E_{H},
$$

where $E_{H}$ is the hydrogen ground state energy. Some average numerical values [18] are $\epsilon_{r} \approx 10$ for inorganic and $\epsilon_{r} \approx 3$ for organic, while the ratio between the effective masses of the electron and the hole can be estimated as $\left(\frac{m}{m_{0}}\right) \approx 0.1$. This leads to $E_{e x} \approx-13.6 \mathrm{meV}$ for inorganic semiconductors and $E_{e x} \approx-151 \mathrm{meV}$ in 
organic semiconductors, leaving us with about one order of magnitude difference in the exciton binding energy.

a

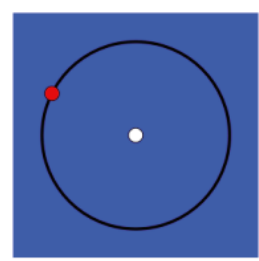

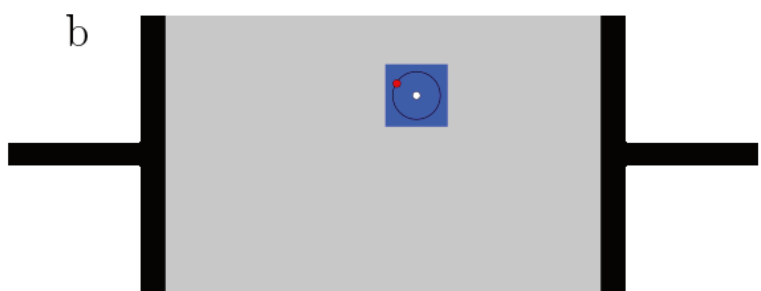

Figure 1.3. Organic semiconductors: a) The excited electron and its related hole remain bonded together forming an hydrogenoid system. b) The so-formed exciton is electrically neutral from a macroscopic point of view and to dissociate it its binding energy has to be overcome.

In summary, inorganic semiconductors have high dielectric constant and low exciton binding energy. The thermal energy at room temperature $\left(k_{B} T=25 \mathrm{meV}\right)$ is sufficient to dissociate the exciton into a positive and negative charge carrier. Indeed in the traditional picture, developed for inorganic semiconductors, when an excitation occurs an electron pass from valence to conduction band, leaving a hole behind. The electron and the hole thus created are considered free ${ }^{1}$ to move in their respective bands (fig. 1.4).

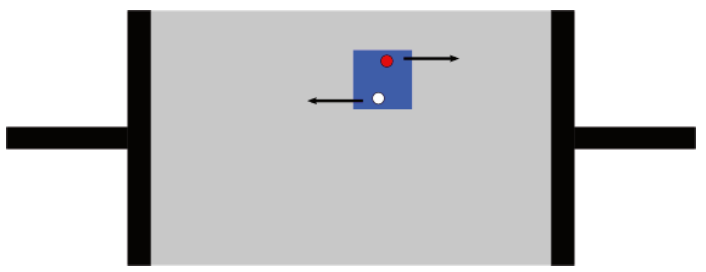

Figure 1.4. In an inorganic semiconductor an excited electron and his related hole are delocalized and can move in the crystal band.

In organic materials, the dissociation of excitons into free charge carriers does not usually occur at room temperature. To overcome this problem, organic solar cells commonly utilize two different materials: an electron donor and an electron acceptor material. The exciton dissociation happens at the interface, where the electron likes to stay on the acceptor, while the hole likes to stay on the donor (fig. 1.5). This process generates a CT state [16,19,20]. Also interfacial dipoles play an important role in helping the charge splitting at the interface [21-24]. In combining donor and acceptor material, care must be taken that the excitons (created in either of these materials) can diffuse to the interface to allow the charge separation process. Due to their short lifetime and low mobility, singlet excitons (usually the most abundant in an OPV) possess a diffusion length of about

\footnotetext{
${ }^{1}$ Free from the mutual attractive interactions.
} 


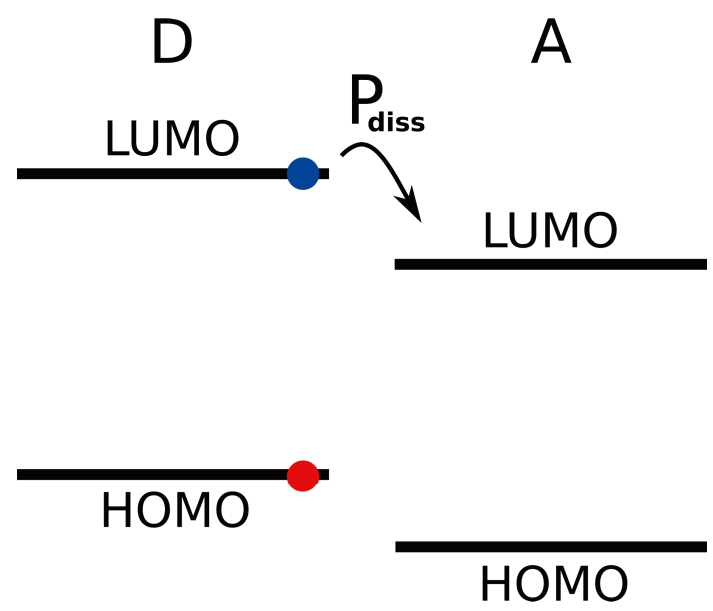

Figure 1.5. Schematic illustration of the exciton splitting mechanism at the interface between donor (D) and acceptor (A).

$10-20 \mathrm{~nm}$ [25]. Thus, anywhere in the active layer, the distance to the interface should be of the order of this average exciton diffusion length. On the other hand, a $20-40 \mathrm{~nm}$ double layer of donor and acceptor materials would let most incident photons to pass through the device freely, without being absorbed. This imposes an important design condition on the interface morphology to enhance the charge generation, as illustrated in [26]. Depending on how the donor and acceptor are mixed together, it is possible to generate different types of junctions and interfaces. The most mentioned in the literature and easy to generate in the lab are the bilayer junction and the bulk heterojunction (BHJ). A bilayer junction contains two layers in between the conductive electrodes (flat interface). This interface favours the free charge carriers transport to the electrodes $\left(\eta_{\text {trans }}\right.$ in fig. 1.2), but only the excitons created in a thin layer around the interface will be able to dissociate (low $\left.\eta_{e x}\right)$. A bulk heterojunction consists of a three-dimensional nanoscale blend of donor and acceptor materials. In this interface the probability of diffusion to the interface $\eta_{e x}$ is very high, but it can result very difficult for the free charge carriers to find a path to the electrodes (low $\left.\eta_{\text {trans }}\right)$. Many other shapes and patterns are interesting efficiency-wise, as for example the interdigitated interface that represents a good compromise between exciton dissociation and free charge carrier transport. Let us notice that in this simplified analysis we neglected the dependence of exciton dissociation and recombination on the interface pattern. Including these processes complicate significantly the picture and the situation has to be studied more in detail. As a last comment, triplet excitons with a longer lifetime and consequently a longer diffusion length, are more likely to diffuse to the interface and split in free charge carriers. Being able to increase the population of triplet excitons in OPV would allow a boost in efficiencies. Research in this area is active through study of singlet-triplet fission [27] or Thermally Activated Delayed Fluorescence [28]. 


\subsection{Modelling Challenges}

As illustrated in section 1.2, we have to date quite a good idea of the main processes involved in organic devices. Even though this is the case, the full understanding of the details of these processes and how they are related to the chemical structure and conformation has not been achieved yet. This lacuna limits our capacity to improve the characteristics of organic devices.

Studies of charge injection and carrier transport in these materials are central to the understanding and improvement of organic devices. The probabilistic nature of the conduction in these materials, joined with their intrinsic disorder, render difficult analytical expressions of the conduction. Analytical studies are mainly limited to molecular crystals using an Hamiltonian coupling electron and phonon dynamics [29-31]. For completeness, the derivation of two such Hamiltonians are reported in section 4. Simulations are the other theoretical tool we can use to understand charge transport in disordered organic materials. They can be done at different levels, but for organic electronics they can be divided in two main important categories. A first category of simulations is based on drift-diffusion models $[26,32,33]$. Some of these studies evidence the significant contribution of morphology to the efficiency of solar cells $[26,33,34]$, but with a detail limited by the classical description employed. The second category aim to a quantum mechanical description of the most relevant processes and is based on Monte Carlo simulations, the natural tool to use in studying the dynamic of competing probabilistic events. This approach, at the price of increasing computational complexity, allows to study more in detail which are the key quantum processes determining the efficiency of an organic solar cell. Furthermore, with the advance of technology we expect devices to become progressively smaller. A model capable of simulating the charge hopping process molecule by molecule is forward-looking for a future nanoscale device modelling. Research in this field can be divided in lattice site description [35-37] and atomistic description $[38,39]$.

We developed a program capable of simulating charge transport in organic materials employing an atomistic description (chapter 5 and papers I-III). The background theory needed to understand the approach used is briefly presented in this manuscript. The morphology of the system is obtained making use of molecular dynamics (MD) simulations, see chapter 2. All calculations regarding the electronic molecular orbitals are performed with well known computational chemistry methods like Hartree-Fock (HF) or density functional theory (DFT), chapter 3. The charge movement is simulated by means of a Kinetic Monte Carlo method (chapter 5) and the probabilities needed to perform the simulation are calculated with Marcus formula (chapter 4). An overview of the results and aim of our research is presented in chapter 6 , followed by three research articles. 


\section{CHAPTER 2}

\section{A Realistic Molecular Arrangement}

Organic materials usually exhibit a disordered amorphous structure. This strongly influences the directionality of the charge transport on a local scale, since the charge carrier will tend to move easier between couples of near $\pi$-stacked molecules. Molecular dynamics (MD) simulations are the appropriate tool to simulate the disorder in organic solar cells. Indeed these methods, based on classical mechanics, allow the simulation of systems of the size of $10-100 \mathrm{~nm}$, a typical domain size of interest in a bulk heterojunction (BHJ) solar cell. A classical potential is used to determine the movement of the atoms. Regarding the study of the dynamics, the atomic movements are governed by Newton equations

$$
\vec{F}=m \frac{d^{2} \vec{r}}{d t^{2}}
$$

where the force is related to the potential energy through

$$
\vec{F}=-\vec{\nabla} U
$$

The potential energy of the system is expressed in terms of atomic interactions, which are divided in bonded and non bonded. Bonded interactions can be expressed in terms of equilibrium points and spring constants and can involve two or more atoms (distances, angles, planar angles). The main non bonded interactions are Coulombic and Van der Waals. Every one of these interactions is parametrized with parameters that depend on the atoms involved. We can for instance think of assigning a different parameter to a couple of carbon atoms depending on their hybridization, i.e. if they are single-, double- or triple-bonded. An analogous reasoning can be carried on for every type of bond in the system. MD simulations are thus carried on upon a specification called a force field. A force field specifies the functional form of the total energy of the system and the set of parameters used 
to calculate it. In the following we will describe some parametrizations made in widely used force fields and we will discuss the algorithm used for MD simulations. In the molecular dynamics exposition we will closely follow reference [40].

\subsection{Force field energetic contributions}

The potential energy function can be decomposed in several terms representing different types of interactions (fig. 2.1). A typical form of the potential energy, as

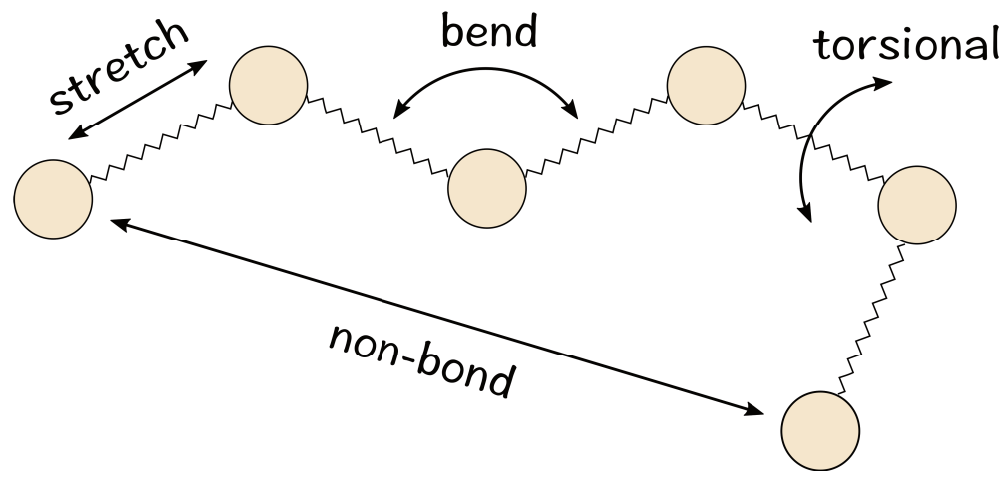

Figure 2.1. Schematic illustration of the different force field contributions. Figure adapted from [41].

implemented also in the OPLS [42,43] force field, is the following

$$
U=U_{\text {stretch }}+U_{\text {bend }}+U_{\text {torsional }}+U_{\text {vanderWaals }}+U_{\text {Coulomb }} .
$$

Stretching and contracting a bond causes an increase in energy. For every couple of bonded atoms, near the equilibrium, the stretching energy can be approximated with a parabola

$$
U_{\text {stretch }}=\frac{1}{2} k_{s}\left(x-x_{0}\right)^{2},
$$

where $k_{s}$ is a force constant determining the strength of the bond, $x$ is the distance between the particles and $x_{0}$ is the equilibrium distance. Also, changing the angle formed among three bonded atoms produces an increase in energy. Near the equilibrium this bending energy can be expressed as

$$
U_{\text {bend }}=\frac{1}{2} k_{\theta}\left(\theta-\theta_{0}\right)^{2},
$$

where $k_{\theta}$ is a force constant, $\theta$ is the angle of the three bonded atoms and $\theta_{0}$ is the equilibrium angle. When a molecule presents a central bond and two atoms are bonded to the opposite sides of this bond, a twist of these two atoms around the central bond causes an increase in energy. The typical expression for this contribution is

$$
U_{\text {torsional }}=V_{n}(1+\cos (n \alpha+\phi)) \text {, }
$$


where $\alpha$ is the planar angle, $n$ is the periodicity of the potential, $V_{n}$ is an energy constant and $\phi$ is a phase shift. One or more of these contributions (for different $n$ ) can be used to express the total torsional potential.

Non bonded interactions are divided in Coulombic interactions representing electrostatic forces and van der Waals interactions including the nuclear repulsions at short distances. Coulombic interactions

$$
U_{\text {Coulomb }}=\frac{q_{1} q_{2}}{4 \pi \epsilon_{0}\left|\vec{r}_{1}-\vec{r}_{2}\right|}
$$

are parametrized through atomic charges for every atom type. Van der Waals interactions are usually approximated through the Lennard-Jones potential, thus are written as

$$
U_{\text {vanderWaals }}=\epsilon\left(\left(\frac{r_{0}}{r}\right)^{12}-2\left(\frac{r_{0}}{r}\right)^{6}\right),
$$

where $\epsilon$ is the depth of the potential well, $r$ is the distance between the particles, and $r_{0}$ is the distance at which the potential reaches its minimum $(-\epsilon)$. For a more complete discussion of the several force field terms we refer the reader to [41].

\subsection{Geometry Optimization}

Once the potential has been calculated, we can look for a stable configuration of the system. This translates in finding the stable equilibrium points of the $3 N-6$ dimensional potential energy surface (PES). This energy surface is usually very complex and this local minima search is achieved with numerical methods such as steepest descent algorithm or Newton/Quasi-Newton methods.

This optimization can be performed also at the quantum level with little changes. In the Born-Oppenheimer approximation (sec. 3.1) the nuclei move in an effective potential generated by the electrons, whose wave functions depend parametrically on the nuclei position. The optimization of the molecular geometry reduces then to the search for the minimum of the effective potential felt by the nuclei. At every step of optimization we can move the nuclei in the potential generated by the electrons, recalculate eigenvalues and eigenfunctions and proceed in this way till convergence. The single point calculations for fixed nuclei can be performed with the desired computational method (chapter 3).

Geometry optimization represents a local exploration of the PES, the optimized structure obtained strongly depend on the starting point. For an extended exploration of the PES, one should turn to Molecular Dynamics.

\subsection{Molecular Dynamics}

Molecular dynamics (MD) is used to simulate the evolution of an ensemble of particles. 'Particles' usually refers to atoms although it may denote any entity for which is possible to define a certain interaction law. In this latter case we talk about coarse grained MD. We will focus in this chapter on atomic MD, more information on coarse grained MD can be found in [40]. Let us assume to have a 
system composed of $N$ atoms, every atom possesses 3 degrees of freedom and the potential energy of the system is expressed as in (2.3). The force acting on the atom $i$ is obtained deriving the potential with respect to the atomic coordinates $\vec{r}_{i}$ and the dynamics of the system can be studied from a generalized $3 \mathrm{~N}$ dimensional newton equation

$$
m_{i} \frac{d^{2} \vec{r}_{i}}{d t^{2}}=-\frac{\partial U}{\partial \vec{r}_{i}} \quad \forall i \in 1 . . N
$$

This set of equations can be integrated numerically with several methods like leapfrog or velocity Verlet [40]. A general algorithm scheme as implemented in the program Gromacs [44-47] is illustrated in figure 2.2. Periodic boundary conditions

\section{THE MD ALGORITHM}

\section{Input initial conditions}

force field specification for the potential $U$ position $\underline{r}$ of all the atoms in the system velocities $\underline{v}$ of all the atoms in the system

$$
\Downarrow
$$

repeat $2,3,4$ for the required number of steps

\section{Compute forces}

the force on any atom

$$
\vec{F}_{i}=-\frac{\partial U}{\partial \vec{r}_{i}}
$$

is the sum of bonded and non-bonded interactions plus external forces if presents

$$
\Downarrow
$$

\section{Update configuration}

the movement of the atoms is simulated by solving numerically Newton's equations of motion

$$
\frac{d^{2} \vec{r}_{i}}{d t^{2}}=-\frac{\vec{F}_{i}}{m_{i}} \quad \forall i \in 1 . . N
$$

4. Output step (if required) write positions, velocilies, energies, temperature, pressure, etc.

Figure 2.2. The MD algorithm as implemented in Gromacs. Figure adapted from ref. [40]. 
are applied to consider infinitely extended materials. At the initial time step, we need to specify the positions and velocities of every atom. If the atomic velocities $\left\{\vec{v}_{i}\right\}$ are not specified they will be generated with random orientation and with modulus $v_{i}$ sampled from a Maxwellian distribution

$$
p\left(v_{i}\right)=4 \pi\left(\frac{m_{i}}{2 \pi k_{B} T}\right)^{3 / 2} v_{i}^{2} \exp \left(-\frac{m_{i} v_{i}^{2}}{2 k_{B} T}\right),
$$

where $k_{B}$ is Boltzmann's constant. The velocities generated in this way have to be corrected before being used in the simulation. First, the velocity of the center of mass is set to 0 . Second, all the velocities are scaled so that the total energy corresponds exactly to $T$; this is done using the equation

$$
E_{k i n}=\sum_{i=1}^{N} \frac{1}{2} m_{i} v_{i}^{2}
$$

and remembering also that

$$
E_{k i n}=\frac{1}{2} N_{f} k_{B} T,
$$

with $N_{f}$ being the total number of degrees of freedom of the system. Equating equations (2.11) and (2.12), a global parameter lambda can be introduced to scale velocities $v_{i} \rightarrow \lambda v_{i}$.

\subsection{Canonical Ensembles}

Since thermodynamic variables are not all independent, only some of them can be fixed during the simulations. Simulations performed following the scheme presented in figure 2.2 will simulate an NVE ensemble, which means that the number of particles, the volume and the energy are kept constant during the dynamic. Usually we are interested in NPT and NVT dynamics since experiments are performed in these conditions, meaning that point 3 in figure 2.2 (Update configuration) has to be modified to generate the right canonical ensemble. The number of particles is always conserved during simulations, while to keep temperature, pressure or volume constants several methods are available in the literature. A brief review of the most used algorithms will be presented in this chapter.

\subsubsection{Berendsen temperature coupling}

A way to control the temperature of the system is by scaling the velocities of every particle with a constant $\lambda$. From (2.11) and (2.12), we obtain

$$
\Delta T=\left(\lambda^{2}-1\right) \frac{\sum_{i} m_{i} v_{i}^{2}}{N_{f} k_{B}}=\left(\lambda^{2}-1\right) T
$$

Berendsen thermostat couples the system to a heat bath at temperature $T_{0}$ imposing

$$
\frac{d T}{d t}=\frac{T_{0}-T}{\tau} .
$$


This ansatz results in an exponential decay of the system towards the desired temperature and $\tau$ is a time constant determining the strength of the coupling. The difference in temperature between two consecutive time steps is

$$
\Delta T=\Delta t \frac{T_{0}-T}{\tau}
$$

where $\Delta t$ is the chosen time step for the integration of the equations of motion. The scaling factor for the velocities can thus be found as

$$
\lambda^{2}=1+\frac{\Delta t}{\tau} \frac{T_{0}-T}{T} .
$$

The value of $\tau$ has to be chosen with care. In the limit of $\tau \rightarrow \infty$ the Berendsen thermostat is inactive and the simulation is evolving as in a microcanonical ensemble. The temperature fluctuations grow until they reach the appropriate value of a microcanonical ensemble, different from those of a canonical ensemble. Very small values of $\tau$ instead will cause unrealistically small temperature fluctuations. Values of $\tau \approx 0.1 p s$ are typically used in MD simulations of condensed-phase systems.

Berendsen thermostat is extremely efficient in relaxing the system to the target temperature [48], but once the equilibrium has been reached there is no guarantee that the fluctuations produced by this method are compatible with a canonical ensemble.

\subsubsection{Nosé-Hoover temperature coupling}

To achieve a smoother control of the temperature, the heat bath should be regarded as an integral part of the system. The Hamiltonian (or Lagrangian) of the system can thus be "augmented" [49] with an extra degree of freedom. This idea appeared at first in a paper by Andersen [50], but it was precised with respect to simulations at constant temperature by Nosé [51] and subsequently developed by Hoover [49].

The system Hamiltonian is extended by introducing an extra degree of freedom $s$ for the thermal reservoir. The interaction between the system of particles and $s$ is expressed with a rescaling of the velocities

$$
\vec{v}_{i}=s \dot{\vec{r}}_{i}
$$

This can be interpreted as an exchange of energy between the system of particles and the heat reservoir. The Hamiltonian for the system is chosen as

$$
H=\sum_{i} s^{2} \frac{\dot{\vec{p}}_{i}^{2}}{2 m_{i}}+U(\underline{r})+\frac{p_{s}^{2}}{2 Q}+N_{f} k_{B} T_{0} \ln s
$$

where $N_{f}$ is the number of degrees of freedom of the system of particles, $T_{0}$ is the reference temperature of the heat bath and $Q$ is a parameter determining the strength of the coupling. $\underline{r}$ has been used to specify the whole set of atomic positions $\left\{\vec{r}_{i}\right\}$. The potential energy associated with the variable $s$ is $N_{f} k_{B} T \ln s$, this form 
of the potential ensures that the canonical ensemble averages are recovered [51]. Introducing the thermodynamic friction coefficient [49]

$$
\zeta=\frac{p_{s}}{Q}
$$

the equations for the evolution of the system can be rewritten as

$$
\begin{aligned}
\dot{\vec{p}}_{i} & =\vec{F}-\zeta \vec{p}_{i} \\
\dot{\zeta} & =\frac{\sum_{i} \frac{\vec{p}_{i}^{2}}{m_{i}}-N_{f} k_{B} T}{Q}
\end{aligned}
$$

where from $(2.20)$ we see how $\zeta$ has indeed the role of a friction coefficient. The value of the parameter $Q$ have to be chosen with some care [48]. If too small the phase space of the system will not be canonical. The tight coupling may cause unphysical high-frequency temperature oscillations that will tend to be off-resonance with the characteristic frequencies of the real system. On the other hand, too large values of $Q$ (loose coupling) cause a poor temperature control and in the limit $Q \rightarrow \infty$ we obtain again the microcanonical ensemble.

\subsubsection{Berendsen pressure coupling}

During an MD simulation the external pressure can be calculated from the tensor virial theorem, presented in Appendix A. The Berendsen algorithm is based on a first order relaxation of the pressure towards a given reference pressure $P_{0}$

$$
\frac{d P}{d t}=\frac{P_{0}-P}{\tau}
$$

Let us apply a rescaling of the coordinates and the box vectors with a factor $\alpha$, i.e.

$$
\frac{d \vec{r}_{i}}{d t}=\vec{v}_{i}-\alpha \vec{r}_{i}
$$

where $\vec{v}_{i}$ is the velocity the particle would have during the simulation without any rescaling. The pressure change is related to the isothermal compressibility $\beta$

$$
\frac{d P}{d t}=-\frac{1}{\beta V} \frac{d V}{d t},
$$

while the volume is changing as

$$
\frac{d V}{d t}=3 \alpha V
$$

these two equations combined gives

$$
\frac{d P}{d t}=-\frac{3 \alpha}{\beta} .
$$


Combining (2.26) and (2.22) gives

$$
\alpha=\frac{\beta\left(P_{0}-P\right)}{3 \tau} .
$$

In its more general formulation (coming from the tensor virial theorem in Appendix A) the coordinates and box vectors are rescaled every $n_{P}$ steps, with the tensor

$$
\mu_{i j}=\delta i j-\frac{n_{P} \Delta t}{3 \tau} \beta_{i j}\left(P_{0 i j}-P_{i j}(t)\right),
$$

where $\beta$ is the compressibility of the system ad $\Delta t$ is the time step. In most cases $\beta$ can be approximated with a scalar; a rough estimate is usually enough since $\beta$ only influences the non-critical time constant of the pressure relaxation without affecting the average pressure. The Berendsen pressure coupling algorithm generates trajectories relaxing to the correct average pressure, but it does not simulate the exact NPT ensemble, "and it is not yet clear exactly what errors this approximation may yield" [40].

\subsubsection{Parrinello-Rahman pressure coupling}

For simulations in which a realistic sampling of the canonical ensemble is needed, to only rescale the volume might not be sufficient. Parrinello and Rahman [52] extended a method proposed by Andersen [50] to let the simulation box also change its shape (very useful for solids undergoing structural transformations, like during crystallization processes).

The vectors of the box $\{\vec{a}, \vec{b}, \vec{c}\}$ can be arranged in a $3 \times 3$ matrix $\mathbf{h}$ whose columns are the latter vectors. The position $\vec{r}_{i}$ of a particle can be written in terms of $\mathbf{h}$ and a column vector $\vec{s}_{i}$ with components $\xi_{i}, \eta_{i}$ and $\zeta_{i}$ as

$$
\vec{r}_{i}=\mathbf{h} \vec{s}_{i}=\xi_{i} \vec{a}+\eta_{i} \vec{b}+\zeta_{i} \vec{c} \quad \text { with } \xi_{i}, \eta_{i}, \zeta_{i} \in[0,1] .
$$

The square of the distance between particle $i$ and $j$ is given by

$$
d_{i j}=\left(\vec{s}_{i}-\vec{s}_{j}\right)^{T} \mathbf{G}\left(\vec{s}_{i}-\vec{s}_{j}\right)
$$

where the metric tensor $\mathbf{G}$ is defined as

$$
\mathbf{G}=\mathbf{h}^{T} \mathbf{h} .
$$

Parrinello-Rahman Lagrangian is augmented with the 9 degrees of freedom coming from $\mathbf{G}$. In case of isotropic pressure it is defined as

$$
\mathcal{L}=\frac{1}{2} \sum_{i} m_{i} \vec{s}_{i}^{T} \mathbf{G} \vec{s}_{i}-U(\underline{r})+\frac{1}{2} W \operatorname{Tr}(\mathbf{G})-p V
$$

where $p$ is the reference pressure and $W$ is a coupling parameter. In the general case, the box vectors evolve with the equations of motion

$$
\frac{d^{2} \mathbf{h}}{d t^{2}}=V \mathbf{W}^{-1} \mathbf{h}^{-T}\left(\mathbf{P}-\mathbf{P}_{0}\right)
$$


where $\mathbf{W}$ now is a matrix of parameters determining the strength of the coupling and how the box can be deformed. The matrices $\mathbf{P}$ and $\mathbf{P}_{0}$ are the current and reference pressures, respectively. The equations of motion for the motion of the particles are [40]

$$
\begin{gathered}
\frac{d^{2} \vec{r}_{i}}{d t^{2}}=\frac{\vec{F}_{i}}{m_{i}}-\mathbf{M} \frac{d \vec{r}_{i}}{d t} \\
\mathbf{M}=\mathbf{h}^{-1}\left(\mathbf{h} \frac{d \mathbf{h}^{T}}{d t}+\frac{d \mathbf{h}}{d t} \mathbf{h}^{T}\right) \mathbf{h}^{-T} .
\end{gathered}
$$

\subsection{The Ising Model}

Ising model has been originally developed to study the spin flip in lattice systems, but in recent times it has also been successfully used to simulate the phase separation processes between a donor and an acceptor material $[36,53]$.

Consider a set of sites $\Lambda$ arranged neatly in forming a d-dimensional lattice. For each lattice site $i \in \Lambda$ there is a discrete variable $\sigma_{i}= \pm 1$, representing a binary property (like spin, or being either of donor or acceptor type). A spin configuration $\sigma=\left\{\sigma_{i}\right\}$ is an assignment of spin to each lattice site.

Any two adjacent sites $i$ and $j$ interacts with an energy $J$. Also, the interaction between the external magnetic field and a lattice site spin results in an energy $h$. The Ising Hamiltonian for such a system is

$$
H(\sigma)=-J \sum_{<i j>} \sigma_{i} \sigma_{j}-h \sum_{j} \sigma_{j}
$$

where the first sum is on nearest neighbours (usually first and second shell). For second shell, the energetic contribution is scaled down by a factor. The configuration probability is given by the Boltzmann distribution

$$
P(\sigma)=\frac{e^{-\beta H(\sigma)}}{Z},
$$

where $\beta=\left(k_{B} T\right)^{-1}$ and $Z$ is the partition function

$$
Z=\sum_{\sigma} e^{-\beta H(\sigma)}
$$

Due to the Boltzmann factor, the spin configurations $\sigma$ with the lowest energies will be favoured. When $h>0, \sigma_{i}=+1$ is favoured, while when $h<0, \sigma_{i}=-1$ is favoured. This means that the spins wants to align with the direction of $h$, as we would expect. Furthermore, when $J>0$ neighbouring spins prefer to be parallel (ferromagnetic model), while when $J<0$ neighbouring spins prefer to be anti-parallel (anti-ferromagnetic model).

The one-dimensional Ising model was solved by Ising in 1925 and present no phase transition [54]. The two-dimensional square lattice Ising model has been solved analytically only in 1944, by Lars Onsager. At low enough temperatures, 
the spins in the 2D Ising model will tend to align themselves even in the absence of the external field (spontaneous magnetization) [55]. Raising the temperature over the critical value $T_{c}$ (depending on the specific material) destroys the spontaneous magnetization. The analytical solution to higher dimensional Ising models is still unknown, but it is possible to study these kind of systems making considerations on their transition temperatures or through Monte Carlo simulations (chapter 5).

Regarding phase separation and interpenetration study of donor (D) and acceptor (A) materials, it is useful to perform Monte Carlo simulations based on the Ising Hamiltonian, setting $h=0$ and allowing the property $\sigma$ to be either D or A. The Ising Hamiltonian for the energy of site $i$ results in

$$
\epsilon_{i}=-\frac{J}{2} \sum_{j}\left(\sigma_{i} \sigma_{j}-1\right),
$$

that gives an energy penalty when $\sigma_{i}=-\sigma_{j}$. At every time step a pair of neighbouring sites with opposite $\sigma$ is selected randomly, and an attempt is made to swap the properties of the two sites. The energy change in the system, $\Delta \epsilon$, caused by swapping the two sites is calculated using (2.39) and the swap is accepted with probability

$$
P(\Delta \epsilon)=\frac{e^{-\Delta \epsilon /\left(k_{B} T\right)}}{1+e^{-\Delta \epsilon /\left(k_{B} T\right)}} .
$$

Starting from a disordered initial condition, performing a MC simulation with this procedure will progressively reduce the disorder of the system and form domains (of exclusively D or A molecules), that will grow bigger till a complete phase separation is happening $[36,53]$. Ising model is a powerful tool to simulate the phase separation
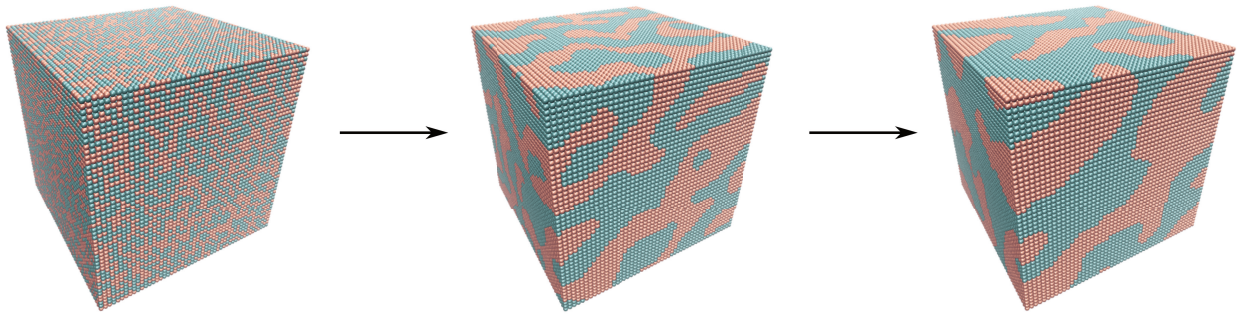

Figure 2.3. Illustration of the Monte Carlo simulation for the phase separation of donor (in pink) and acceptor (in cyan) molecules, based on the Ising Hamiltonian. From left to right we find the initial state and the state of the system after 300 and 1000 Monte Carlo steps.

of the donor and acceptor materials (fig. 2.3). This task would be prohibitive employing MD simulations due to the long timescale of the phenomenon. Different degrees of interpenetration can be obtained along the Monte Carlo trajectory based on the Ising Hamiltonian. Once the desired interpenetration has been achieved, the volume of every cell of the lattice can be filled with molecules of the right type, D or A. The morphology thus obtained can be used as a starting point for an MD simulation. 


\section{CHAPTER 3}

\section{Molecular Orbitals Theory}

A charge carrier hops from one molecule to another in a series of probabilistic events as discussed in chapter 1 . When sitting on a molecule it occupies a particular molecular level and the shape and extension of this latter will strongly affect the charge transport. Furthermore the energies and the wave functions of the molecular orbitals will be affected by the molecular conformation in the bulk. All these aspects can be taken into account in a quantum mechanical description, the Schrödinger equation of the molecule contains in principle all the informations needed. Its explicit analytical solution is however impossible in the majority of cases and the development of physical approximations becomes of central importance for obtaining a solution. With the development of modern computers, the popularity of the computational methods of Quantum Chemistry is rapidly increasing, allowing us nowadays to treat one or few molecules with a fully quantum description. To understand more in detail advantages and limitations of some of the most popular Quantum Chemistry methods used in the literature, a brief review is presented in this chapter. For HF and DFT, the exposition will closely follow the approach of Jensen [41].

\subsection{Born-Oppenheimer Approximation}

Without considering magnetic interactions, the Schrödinger equation for the molecule can be written as

$$
\left(T_{e}+V_{e n}+V_{e e}+T_{n}+V_{n n}\right) \Psi(\underline{r}, \underline{R})=E_{M} \Psi(\underline{r}, \underline{R})
$$


where the various terms represent

$$
\begin{array}{rlr}
T_{e} & =-\sum_{i} \frac{\hbar^{2}}{2 m_{i}} \nabla_{i}^{2} & \text { the kinetic energy of the electrons } \\
T_{n} & =-\sum_{\alpha} \frac{\hbar^{2}}{2 M_{\alpha}} \nabla_{\alpha}^{2} & \text { the kinetic energy of the nuclei } \\
V_{e n} & =-\sum_{\alpha, i} \frac{Z_{\alpha} e^{2}}{\left|\vec{r}_{i}-\vec{R}_{\alpha}\right|} & \text { electrons-nuclei Coulombic attraction } \\
V_{e e} & =\sum_{i<j} \frac{e^{2}}{\left|\vec{r}_{i}-\vec{r}_{j}\right|} & \text { electronic Coulombic repulsion } \\
V_{n n} & =\sum_{\alpha<\beta} \frac{Z_{\alpha} Z_{\beta} e^{2}}{\left|\vec{R}_{\alpha}-\vec{R}_{\beta}\right|} & \text { nuclei Coulombic repulsion. }
\end{array}
$$

With $\underline{r}$ and $\underline{R}$ we have identified the set of coordinates of the electrons $\left\{\vec{r}_{i}\right\}$ and of the nuclei $\left\{\vec{R}_{\alpha}\right\}$.

The Born-Oppenheimer approximation relies on the disparity of mass (and thus velocity) of nuclei and electrons. As a result of this imbalance, in the time in which the nuclei moves a little, the electrons have time to settle in the molecular orbitals (MO) at that nuclear configuration. That is, the electrons follow adiabatically the nuclei, in a succession of states of equilibrium $\psi_{e}(\underline{r}, \underline{R})$, solutions of a stationary Schrödinger equation with fixed nuclei. The electronic wave functions are therefore considered only parametrically dependent on the nuclei position. We can factorize the wave function of the system as

$$
\Psi(\underline{r}, \underline{R})=\psi_{e}(\underline{r}, \underline{R}) \phi_{n}(\underline{R})
$$

where $\phi_{n}$ depends only on the nuclear positions and $\psi_{e}$ describes the electronic states in a fixed nuclei configuration. The electronic Schrödinger equation can be written as

$$
\left(T_{e}+V_{e n}+V_{e e}\right) \psi_{e}(\underline{r}, \underline{R})=E_{e}(\underline{R}) \psi_{e}(\underline{r}, \underline{R})
$$

with the $\underline{R}$ coordinates constant. $H_{e}(\underline{R})=T_{e}+V_{e n}+V_{e e}$ is the Hamiltonian of the electronic system and $E_{e}(\underline{R})$ is the eigenvalue of the wave function $\psi_{e}(\underline{r}, \underline{R})$. Substituting (3.7) and (3.8) in the general equation (3.1) and having the operator $T_{n}$ acting only on the nuclear wave function, we obtain

$$
\left(T_{n}+V_{n n}+E_{e}(\underline{R})\right) \phi_{n}(\underline{R})=E_{M} \phi_{n}(\underline{R}) .
$$

$H_{n}=T_{n}+V_{n n}+E_{e}(\underline{R})$ is the Hamiltonian of the nuclear system, with eigenvalue $E_{M}$ and eigenfunction $\phi_{n}(\underline{R})$. The nuclei are subject to the effective potential $V_{e f f}=V_{n n}+E_{e}(\underline{R})$, sum of the repulsive potential $V_{n n}$ and the attractive one given by the electronic contribution $E_{e}(\underline{R})$.

The original Schrödinger equation for the molecule (3.1) has been decoupled in two equations, one for the electrons and one for the nuclei. To achieve this results 
we had the operator $T_{n}$ act only on the nuclear wave function $\phi_{n}(\underline{R})$. In reality the action of $T_{n}$ on $(3.7)$ is

$$
\begin{aligned}
T_{n}\left(\psi_{e}(\underline{r}, \underline{R}) \phi_{n}(\underline{R})\right) & =-\sum_{\alpha} \frac{\hbar^{2}}{2 M_{\alpha}} \nabla_{\alpha}^{2}\left(\psi_{e} \phi_{n}\right)= \\
= & -\sum_{\alpha} \frac{\hbar^{2}}{2 M_{\alpha}}\left(\psi_{e} \nabla_{\alpha}^{2} \phi_{n}+2 \nabla_{\alpha} \psi_{e} \cdot \nabla_{\alpha} \phi_{n}+\phi_{n} \nabla_{\alpha}^{2} \psi_{e}\right),
\end{aligned}
$$

so that in summary Born-Oppenheimer approximation consists in neglecting the last two terms of (3.10). For many systems of interests this is a reasonable approximations. For other systems this is not the case, and transitions between two Born-Oppenheimer surfaces are happening through coupling of vibrational levels.

\subsection{Symmetrization and Antisymmetrization Op- erators}

Assume the state of a single particle $u_{\alpha}$ is a ket in the Hilbert space $\mathcal{H}$, the system composed of $N$ identical particles live in the Cartesian product $\mathcal{H}^{N}$. A simple composition of such one-particle states can be

$$
|\psi\rangle=\left|u_{\alpha_{1}}^{(1)}, u_{\alpha_{2}}^{(2)}, \ldots, u_{\alpha_{N}}^{(N)}\right\rangle=\left|u_{\alpha_{1}}^{(1)}\right\rangle \otimes\left|u_{\alpha_{2}}^{(2)}\right\rangle \otimes \cdots \otimes\left|u_{\alpha_{N}}^{(N)}\right\rangle .
$$

A transposition of two particles $i$ and $j$ (with $i>j$ ) is performed by the operator

$$
T_{i j}\left|u_{\alpha_{1}}^{(1)}, \ldots u_{\alpha_{i}}^{(i)}, \ldots u_{\alpha_{j}}^{(j)}, \ldots u_{\alpha_{N}}^{(N)}\right\rangle=\left|u_{\alpha_{1}}^{(1)}, \ldots u_{\alpha_{j}}^{(i)}, \ldots u_{\alpha_{i}}^{(j)}, \ldots u_{\alpha_{N}}^{(N)}\right\rangle
$$

that is hermitian and unitary as can be easily checked. The operator $P_{\sigma}$ performing a permutation $\sigma$ of the set of identical particles

$$
P_{\sigma}\left|u_{\alpha_{1}}^{(1)}, u_{\alpha_{2}}^{(2)}, \ldots, u_{\alpha_{N}}^{(N)}\right\rangle=\left|u_{\alpha_{\sigma(1)}}^{(1)}, u_{\alpha_{\sigma(2)}}^{(2)}, \ldots, u_{\alpha_{\sigma(N)}}^{(N)}\right\rangle,
$$

is a composition of transposition $P_{\sigma}=T_{1} \circ T_{2} \circ \cdots$. Thus a permutation $P_{\sigma}$ is unitary, but in general not hermitian because the transpositions $\left\{T_{i j}\right\}$ does not always commute with each other ${ }^{1}$. Let us introduce the symmetrization and antisymmetrization operators, which generates the states for bosons and fermions respectively

$$
\begin{aligned}
& S=\frac{1}{N !} \sum_{\sigma} P_{\sigma} \\
& A=\frac{1}{N !} \sum_{\sigma} \operatorname{sgn} \sigma P_{\sigma} .
\end{aligned}
$$

\footnotetext{
${ }^{1}$ Let $P=T_{1} T_{2}$, if $T_{1}$ and $T_{2}$ are unitary the inverse operator of $P$ is $P^{-1}=T_{2}^{-1} T_{1}^{-1}=T_{2}^{\dagger} T_{1}^{\dagger}=P^{\dagger}$,

thus $P$ is unitary. Instead if $T_{1}$ and $T_{2}$ are hermitian, we obtain

$$
P^{\dagger}=T_{2}^{\dagger} T_{1}^{\dagger}=T_{2} T_{1}
$$

that is equal to $P$ only if $\left[T_{1}, T_{2}\right]=0$, thus $P$ is hermitian only in that case.
} 
Using the properties

$$
\begin{aligned}
& P_{\sigma_{0}} S=\frac{1}{N !} \sum_{\sigma} P_{\sigma_{0}} P_{\sigma}=\frac{1}{N !} \sum_{\sigma^{\prime}} P_{\sigma^{\prime}}=S \\
& P_{\sigma_{0}} A=\operatorname{sgn}_{0} A
\end{aligned}
$$

we obtain

$$
\begin{aligned}
& S S=\frac{1}{N !} \sum_{\sigma} P_{\sigma} S=S \\
& A A=\frac{1}{N !} \sum_{\sigma} \operatorname{sgn} \sigma P_{\sigma} A=A,
\end{aligned}
$$

i.e. operators $S$ and $A$ are idempotent. $S$ and $A$ are unitary and idempotent, it follows that they are projection operators; they identify $\mathcal{H}_{S}$ and $\mathcal{H}_{A}$, two subspaces of $\mathcal{H}^{N}$. The subspace $\mathcal{H}_{S}$ contains all the symmetric states of $\mathcal{H}^{N}$, while the subspace $\mathcal{H}_{A}$ contains all the antisymmetric ones. Furthermore, given that

$$
A S=S A=\frac{1}{N !} \sum_{\sigma} \operatorname{sgn} \sigma P_{\sigma} S=\frac{1}{N !} S \sum_{\sigma} \operatorname{sgn} \sigma=0
$$

these subspaces are also orthogonal $\mathcal{H}_{S} \perp \mathcal{H}_{A}$.

\subsection{Hartree-Fock}

The electronic time-independent Schrödinger equation obtained after invoking the Born-Oppenheimer approximation can be solved exactly only in few very simple cases. To generate approximate solutions we will employ the variational principle, which states that any approximate wave function has an energy above or equal to the exact energy; with the equality holding only if the approximate function is the exact wave function. We can construct a trial wave function $\psi_{\gamma}$ containing a certain number of parameters $\gamma$, the "best" function of this given form is the $\psi_{\gamma}^{*}$ obtained by minimizing the energy

$$
E=\left\langle\psi_{\gamma}|H| \psi_{\gamma}\right\rangle
$$

as a function of the parameters $\gamma$.

Hartree-Fock theory starts from the ansatz that the electronic wave function has the form of a single Slater determinant

$$
|\psi\rangle=A\left|u_{\alpha_{1}}^{(1)}, u_{\alpha_{2}}^{(2)}, \ldots, u_{\alpha_{N}}^{(N)}\right\rangle .
$$

The electronic Hamiltonian can be written in terms of zero-, one- and two-electron terms

$$
H=h_{0}+\sum_{i} h_{i}+\sum_{\substack{i, j \\ i<j}} g_{i j}
$$


$h_{0}$ is and additive constant given by the repulsion of the nuclei at the particular position considered,

$$
h_{i}=-\frac{1}{2} \nabla_{i}^{2}-\sum_{\alpha} \frac{Z_{\alpha} e^{2}}{\left|\vec{r}_{i}-\vec{R}_{\alpha}\right|}
$$

is the electron-nuclei attraction and

$$
g_{i j}=\frac{e^{2}}{\left|\vec{r}_{i}-\vec{r}_{j}\right|}
$$

describes the interaction between two electrons. Using the concepts of section 3.2 the energy of (3.19) can be found as

$$
\begin{aligned}
E[\psi]=\langle\psi|H| \psi\rangle= & \\
& \sum_{i}\left\langle\varphi_{i}|\hat{h}| \varphi_{i}\right\rangle+\frac{1}{2} \sum_{i, j}\left(\left\langle\varphi_{i} \varphi_{j}|\hat{g}| \varphi_{i} \varphi_{j}\right\rangle-\left\langle\varphi_{i} \varphi_{j}|\hat{g}| \varphi_{j} \varphi_{i}\right\rangle\right)
\end{aligned}
$$

where we have introduced the one electron operator

$$
\hat{h}=-\frac{1}{2} \nabla^{2}-\sum_{\alpha} \frac{Z_{\alpha} e^{2}}{\left|\vec{r}-\vec{R}_{\alpha}\right|}
$$

and the two electron operator

$$
\hat{g}=\frac{e^{2}}{\left|\vec{r}_{1}-\vec{r}_{2}\right|} .
$$

Let us introduce the Coulomb operator $J_{i}$ and the exchange operator $K_{i}$, defined as

$$
\begin{aligned}
\hat{J}_{i} f(1) & =\int \frac{e \varphi_{i}^{*}(2) \varphi_{i}(2)}{r_{12}} d V_{2} f(1) \\
\hat{K}_{i} f(1) & =\int \frac{e \varphi_{i}^{*}(2) f(2)}{r_{12}} d V_{2} \varphi_{i}(1)
\end{aligned}
$$

and the total Coulomb and exchange operators

$$
\begin{gathered}
\hat{J}=\sum_{i} \hat{J}_{i} \\
\hat{K}=\sum_{i} \hat{K}_{i} .
\end{gathered}
$$

Equation (3.23) can thus be rewritten as

$$
E[\psi]=\sum_{i}\left\langle\varphi_{i}|\hat{h}| \varphi_{i}\right\rangle+\frac{1}{2} \sum_{i}\left(\left\langle\varphi_{i}|(\hat{J}-\hat{K})| \varphi_{i}\right\rangle\right) .
$$

The objective is now to determine a set of MOs that makes the energy a minimum, while the variation of such orbitals is such that the MOs remain orthogonal and 
normalized. This is a constrained optimization, and can be solved by means of Lagrange multipliers, i.e. finding the stationary point of the Lagrangian function

$$
L[\psi]=E[\psi]-\sum_{i j} \lambda_{i j}\left(\left\langle\varphi_{i} \mid \varphi_{j}\right\rangle-\delta_{i j}\right)
$$

From the (3.30) the variation of the energy with respect to the functional variation of the MOs can be written as

$$
\delta E[\psi]=\sum_{i}\left\langle\delta \varphi_{i}|\hat{h}| \varphi_{i}\right\rangle+\frac{1}{2} \sum_{i}\left(\left\langle\delta \varphi_{i}|(\hat{J}-\hat{K})| \varphi_{i}\right\rangle\right)+c . c .=\sum_{i}\left\langle\delta \varphi_{i}|\hat{F}| \varphi_{i}\right\rangle+c . c .
$$

In this equation we have introduced the Fock operator

$$
\hat{F}=\hat{h}+\hat{J}-\hat{K},
$$

this operator can be regarded as an effective one-electron energy operator, including the attractive potential of the nuclei as well as an average of repulsive Coulombic and exchange interactions of the other electrons (with $j \neq i$ ). In particular the exchange interactions enter into the picture as a purely quantum mechanical effect coming from the Slater determinant form of the wave function for identical fermions. The variation of the Lagrangian (3.31) can be written as

$$
\begin{aligned}
& \delta L[\psi]=\sum_{i}\left\langle\delta \varphi_{i}|\hat{F}| \varphi_{i}\right\rangle-\sum_{i j} \lambda_{i j}\left\langle\delta \varphi_{i} \mid \varphi_{j}\right\rangle+ \\
& \sum_{i}\left\langle\delta \varphi_{i}|\hat{F}| \varphi_{i}\right\rangle^{*}-\sum_{i j} \lambda_{i j}\left\langle\delta \varphi_{j} \mid \varphi_{i}\right\rangle^{*}=0 .
\end{aligned}
$$

Since the variations $\left|\delta \varphi_{i}\right\rangle$ and $\left|\delta \varphi_{i}\right\rangle^{*}$ can be chosen independently, equation (3.34) can be split into a set of two equations

$$
\begin{gathered}
\sum_{i}\left\langle\delta \varphi_{i}|\hat{F}| \varphi_{i}\right\rangle-\sum_{i j} \lambda_{i j}\left\langle\delta \varphi_{i} \mid \varphi_{j}\right\rangle=0 \\
\sum_{i}\left\langle\delta \varphi_{i}|\hat{F}| \varphi_{i}\right\rangle^{*}-\sum_{i j} \lambda_{i j}\left\langle\delta \varphi_{j} \mid \varphi_{i}\right\rangle^{*}=0 .
\end{gathered}
$$

Taking the complex conjugate of (3.36) and subtracting it to (3.35), we obtain

$$
\sum_{i j}\left(\lambda_{i j}-\lambda_{j i}^{*}\right)\left\langle\delta \varphi_{i} \mid \varphi_{j}\right\rangle=0
$$

that means that the matrix of the Lagrange multipliers $\lambda_{i j}$ is Hermitian. From (3.35) the Hartree-Fock (HF) equations can be written as

$$
\hat{F}\left|\varphi_{i}\right\rangle=\sum_{i j} \lambda_{i j}\left|\varphi_{j}\right\rangle
$$

but since $\lambda_{i j}$ is Hermitian, it exists a transformation of the MOs such that

$$
\hat{F}\left|\varphi_{i}\right\rangle=\epsilon_{i}\left|\varphi_{i}\right\rangle
$$


where we have called again $\left\{\varphi_{i}\right\}$ the set of transformed orbitals.

For the numerical solution of the HF equations, the unknown MOs are expressed in terms of a set of known functions, the basis set. The basis set has to be chosen carefully, in a way that functions of the basis are somehow able to capture the physics of the problem. Employing a basis set allows to pass from an infinite dimensional problem (in the Hilbert space $L^{2}$ ) to a finite dimensional algebraic problem. The behaviour of such functions should be able to capture the physics of the problem, allowing to approximate better the real solution. Every molecular orbital is expanded as a linear combination of atomic orbitals (LCAO)

$$
\varphi_{i}(r)=\sum_{a=1}^{M} C_{a i} \chi_{a}(r)
$$

where the $\left\{\chi_{a}\right\}$ are forming a basis of dimension $M$. This leads to the Roothan-Hall $(\mathrm{RH})$ equations, a generalized eigenvalue problem for an $\mathrm{M}$ x M matrix

$$
F C=S C \epsilon
$$

where $F_{a b}=\left\langle\chi_{a}|\hat{F}| \chi_{b}\right\rangle$ and $S_{a b}=\left\langle\chi_{a} \mid \chi_{b}\right\rangle$. The Fock matrix results

$$
\begin{array}{r}
\left\langle\chi_{a}|\hat{F}| \chi_{b}\right\rangle=\left\langle\chi_{a}|\hat{h}| \chi_{b}\right\rangle+\sum_{j}^{o c c . M O s}\left\langle\chi_{a}\left|\hat{J}_{j}-\hat{K}_{j}\right| \chi_{b}\right\rangle \\
=\left\langle\chi_{a}|\hat{h}| \chi_{b}\right\rangle+\sum_{j}^{o c c . M O s}\left\langle\chi_{a} \varphi_{j}|\hat{g}| \chi_{b} \varphi_{j}\right\rangle-\left\langle\chi_{a} \varphi_{j}|\hat{g}| \varphi_{j} \chi_{b}\right\rangle \\
=\left\langle\chi_{a}|\hat{h}| \chi_{b}\right\rangle+\sum_{c, d}^{M} D_{c d}\left(\left\langle\chi_{a} \chi_{c}|\hat{g}| \chi_{b} \chi_{d}\right\rangle-\left\langle\chi_{a} \chi_{c}|\hat{g}| \chi_{d} \chi_{b}\right\rangle\right)
\end{array}
$$

it is depending only on the occupied molecular orbitals through the density matrix

$$
D_{a b}=\sum_{j}^{o c c . M O s} C_{a j} C_{b j}
$$

and the fourth order tensor

$$
G_{a b c d}=\left\langle\chi_{a} \chi_{c}|\hat{g}| \chi_{b} \chi_{d}\right\rangle-\left\langle\chi_{a} \chi_{c}|\hat{g}| \chi_{d} \chi_{b}\right\rangle
$$

involving the two-electron integrals. The solution to the $\mathrm{RH}$ equations (3.41) are $\mathrm{M}$ eigenvalues $\epsilon_{i}$ with their associated set of coefficients $\left\{C_{i 1}, C_{i 2}, \ldots, C_{i M}\right\}, i=$ $1, \ldots, M$. Each index i represents an MO giving us as a solution $\mathrm{M}$ molecular orbitals; and if $\mathrm{N}$ is the number of electrons, only $\mathrm{N} / 2$ of them are occupied (in the closed shell system). Once the solution to the RH equations has been obtained, a new Fock matrix can be constructed. This process is iteratively repeated in a self-consistent field (SCF) calculation till convergence is reached (fig. 3.1). It is important to notice that the occupied orbitals are variationally optimized, because 


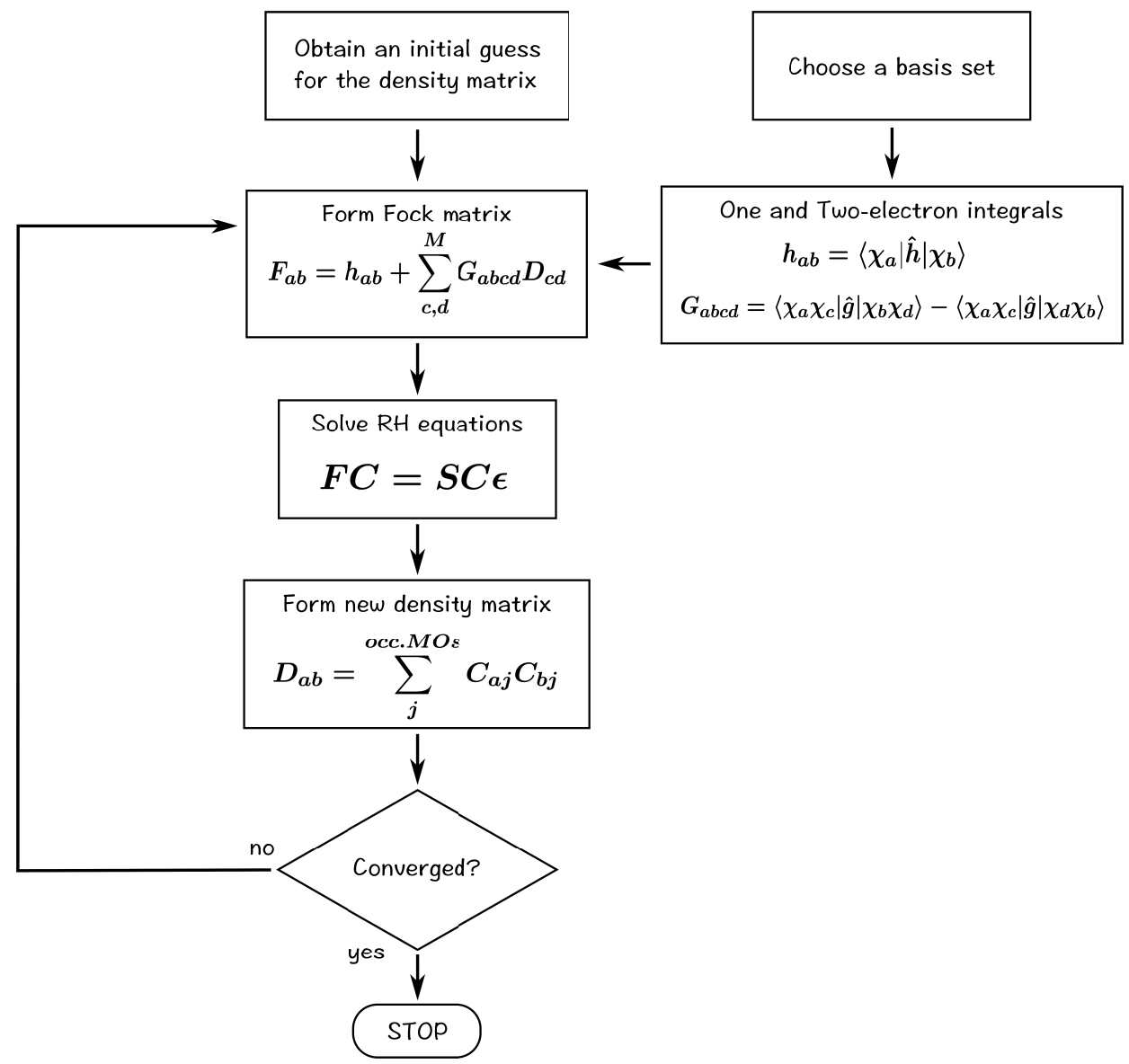

Figure 3.1. The SCF procedure for the HF method.

they do contribute to the total energy and then to the Fock operator at a particular step of the SCF cycle. It is not clear instead how reliable are eigenvalues and eigenfunctions of unoccupied orbitals.

In the solution of the Hartree-Fock method the electrons are sitting on effective orbitals coming from an average interaction. If the basis set is sufficiently large, the HF solution accounts for $\sim 99 \%$ of the total energy. As a matter of fact, the remaining $\sim 1 \%$ is often very important for describing chemical phenomena [41]. The missing piece in the HF picture is electronic correlation. In an attempt to account for correlation we can use as an ansatz a linear combination of Slater determinants corresponding to excited state configurations. This gives origin to the so called post Hartree-Fock methods, such as configuration interaction (CI), coupled-cluster (CC) and Møller-Plesset perturbation theory (MP). These approaches allows for a systematic increase of accuracy, but the computational cost 
increases dramatically with the number of excited levels considered.

\subsection{Semi empirical Methods}

The cost of performing an HF calculation scales formally as the fourth power of the number of basis functions (the cost of calculating the two-electron integrals needed to calculate the matrix $F_{a b}$, at every step). The first step in reducing the computational problem is to consider only the valence electrons explicitly, while accounting for the core electrons by reducing the nuclear charge (effective screening). A minimal basis set can be used for the valence electrons states. For the hydrogen one $s$ function is considered, while atoms in the second and third row of the periodic table are described with four basis functions for $s$ and $p$ orbitals. Most semi-empirical methods use only s- and p- functions, and Slater type orbitals as basis functions [41].

The central assumption of semi-empirical methods is the Zero Differential Overlap (ZDO) approximation, which neglects all the products of basis functions located on different atoms. The integrals that are different from zero are considered as parameters and are determined based on theoretical calculations or experimental data. This procedure is also known in literature as parametrization of a particular semi-empirical method.

\subsubsection{NDDO}

Employing the ZDO approximation leads to the Neglect of Diatomic Differential Overlap (NDDO) method. The three- and four-center integrals vanish. The overlap matrix $\mathrm{S}$ is reduced to

$$
S_{\mu \nu}=\langle\mu \mid \nu\rangle=\delta_{\mu \nu}
$$

where $\mu$ and $\nu$ are two atomic orbitals. The one-electron integrals are

$$
\begin{aligned}
& \left\langle\mu_{A}|\hat{h}| \nu_{A}\right\rangle=\left\langle\mu_{A}\left|-\frac{1}{2} \nabla^{2}+V_{A}\right| \nu_{A}\right\rangle+\sum_{a \neq A}\left\langle\mu_{A}\left|V_{a}\right| \nu_{A}\right\rangle \\
& \left\langle\mu_{A}|\hat{h}| \nu_{B}\right\rangle=\left\langle\mu_{A}\left|-\frac{1}{2} \nabla^{2}+V_{A}+V_{B}\right| \nu_{A}\right\rangle
\end{aligned}
$$

where $V_{A}$ is the atomic potential (nucleus and core electrons) of the atom $A$ and $\mu_{A}$ and $\nu_{A}$ are atomic orbitals of the atom $A$. Due to the orthogonality of the atomic orbitals $\left\langle\mu_{A}\left|-\frac{1}{2} \nabla^{2}+V_{A}\right| \nu_{A}\right\rangle$ is 0 unless $\mu=\nu$. The two-electron integrals are approximated as

$$
\left\langle\mu_{A} \nu_{B}|\hat{g}| \lambda_{C} \sigma_{D}\right\rangle=\delta_{A C} \delta_{B D}\left\langle\mu_{A} \nu_{B}|\hat{g}| \lambda_{A} \sigma_{B}\right\rangle .
$$

\subsubsection{INDO}

The Intermediate Neglect of Differential Overlap (INDO) [56-58] approximation neglects all two-center two-electron integrals that are not of the Coulomb type, in addition to those neglected by the NDDO approximations. In order to preserve 
rotational invariance, i.e. the total energy should be independent of a rotation of the coordinate system, the integrals of the form $\left\langle\mu_{A}\left|V_{a}\right| \nu_{A}\right\rangle$ and $\left\langle\mu_{A} \nu_{B}|\hat{g}| \lambda_{A} \sigma_{B}\right\rangle$ must be taken independent of the orbital types (i.e. an integral involving a p-orbital must be the same as with an s-orbital) [56]. This leads to the INDO method, involving the following approximations in addition to those made by NDDO. One-electron integrals become

$$
\begin{aligned}
& \left\langle\mu_{A}|h| \mu_{A}\right\rangle=\left\langle\mu_{A}\left|-\frac{1}{2} \nabla^{2}+V_{A}\right| \mu_{A}\right\rangle+\sum_{a \neq A}\left\langle\mu_{A}\left|V_{a}\right| \mu_{A}\right\rangle \\
& \left\langle\mu_{A}|h| \nu_{A}\right\rangle=0
\end{aligned}
$$

Two-electron integrals are

$$
\left\langle\mu_{A} \nu_{B}|\hat{g}| \lambda_{A} \sigma_{B}\right\rangle=\delta_{\mu \lambda} \delta_{\nu \sigma}\left\langle\mu_{A} \nu_{B}|\hat{g}| \mu_{A} \nu_{B}\right\rangle \text {. }
$$

The non zero two-electron integrals are usually denoted by $\gamma$

$$
\begin{aligned}
\left\langle\mu_{A} \nu_{A}|\hat{g}| \mu_{A} \nu_{A}\right\rangle & =\left\langle\mu_{A} \mu_{A}|\hat{g}| \mu_{A} \mu_{A}\right\rangle=\gamma_{A A} \\
\left\langle\mu_{A} \nu_{B}|\hat{g}| \mu_{A} \nu_{B}\right\rangle & =\gamma_{A B} .
\end{aligned}
$$

\subsubsection{ZINDO}

ZINDO [59] is a parametrization of INDO that covers a wide range of the periodic table, even including the rare earth elements.

\subsection{Density Functional Theory}

The density functional theory (DFT) allows us to calculate the ground state properties of a system without dealing (at least in principle) with the manyelectron wave function $|\psi\rangle$. In DFT the main quantity of interest is the electronic density

$$
n(\vec{r})=N \sum_{\sigma_{1}} \int d \vec{x}_{2} \ldots \int d \vec{x}_{n}\left|\psi\left(\vec{x}_{1}, \vec{x}_{2}, \ldots, \vec{x}_{N}\right)\right|^{2}
$$

where in this notation $\vec{x}_{i}$ represents all the degrees of freedom of particle $i$, position and spin $\vec{x}_{i}=\left(\vec{r}_{i}, \sigma_{i}\right)$. In the Schrödinger equation for the electrons in BornOppenheimer approximation (3.8), the Coulomb potential arising from the nuclei is treated as a static external potential

$$
\hat{V}_{e x t}=-\sum_{\alpha, i} \frac{Z_{\alpha} e^{2}}{\left|\vec{r}_{i}-\vec{R}_{\alpha}\right|}
$$

while the remaining part of the Hamiltonian is

$$
\hat{F}_{H K}=\hat{T}_{e}+\hat{V}_{e e}
$$

such that $H=\hat{F}_{H K}+\hat{V}_{e x t}$

$$
\left(\hat{F}_{H K}+\hat{V}_{e x t}\right)|\psi\rangle=E|\psi\rangle .
$$


Since $\hat{F}$ is the same for all $N$-electron systems, the Hamiltonian and hence the ground-state $\left|\psi_{0}\right\rangle$, are completely determined by $N$ and $\hat{V}_{\text {ext }}$.

The average energy is

$$
E=\left\langle\psi\left|\hat{F}_{H K}\right| \psi\right\rangle+\left\langle\psi\left|\hat{V}_{\text {ext }}\right| \psi\right\rangle
$$

where the energy of the external potential, as in Thomas-Fermi theory, can be expressed as

$$
\left\langle\psi\left|\hat{V}_{e x t}\right| \psi\right\rangle=\int v(\vec{r}) n(\vec{r}) d \vec{r}=V_{e x t}[n] .
$$

Density-functional theory is based on two fundamental theorems by Hohenberg and Kohn [60,61].

Theorem 3.1 The external potential $v(\vec{r})$ is uniquely determined, up to a constant, by the ground state electron density $n_{0}(\vec{r})$.

As a consequence, all the properties of the system are determined by the electronic ground state density. Indeed, since $n_{0}(\vec{r})$ determines both the external potential $v(\vec{r})$ and the number of electrons $N=\int d \vec{r} n_{0}(\vec{r})$, it also characterize unambiguously the Hamiltonian $H$ and thus implicitly all the properties derivable from $H$ through the solution of the time-independent or time-dependent Schrödinger equation, like the wave functions $|\psi\rangle$. Furthermore, it exists a functional $E[n]$, that expresses the energy in terms of the electron density, for any particular $v(\vec{r})$

$$
E[n]=T_{e}[n]+V_{e e}[n]+V_{e x t}[n]=F_{H K}[n]+V_{e x t}[n] .
$$

$F_{H K}[n]$ is a universal functional, completely independent on the external potential $v(\vec{r})$ and it is thus the same for any set of $N$ electrons.

Theorem 3.2 The ground state energy of the system is the global minimum $E_{0}=E\left[n_{0}\right]$ of the functional $E[n]$, and $n_{0}$ is the electronic density of the ground state.

The problem of determining the ground state of a Schrödinger equation with $3 N$ degrees of freedom can be translated in determining the minimum of a functional $E[n]$, where $n$ is a function of 3 variables. This apparently trivial problem is in practice very difficult since the form of the functional $E[n]$, and in particular of $F_{H K}[n]$ is unknown. $F_{H K}[n]$ contains the functional for the kinetic energy $T[n]$ and for the electron-electron interaction $V_{e e}[n]$. The explicit form of both these functional is unknown. However, it is usually common practice to extract the classical part of the electronic repulsion (also called Hartree energy, denoted as $\left.E_{H}\right)$, writing

$$
V_{e e}[n]=\frac{1}{2} \iint \frac{n\left(\vec{r}_{1}\right) n\left(\vec{r}_{2}\right)}{r_{12}} d \vec{r}_{1} d \vec{r}_{2}+E_{x c}[n]=E_{H}[n]+E_{x c}[n]
$$

thus defining a new functional $E_{x c}[n]$ containing the non-classical part of the electron-electron interactions, usually identified as exchange and correlation contributions. To determine $T[n]$ and $E_{x c}[n]$ represents the major challenge of DFT. 


\subsubsection{Kohn-Sham Scheme}

The idea is to replace the system of interacting particles in the external potential $v(\vec{r})$ with a system of non-interacting particles in some other external potential $v_{s}(\vec{r})$

$$
\left(-\frac{\hbar^{2}}{2 m} \nabla^{2}+v_{s}(\vec{r})\right) \varphi_{i}(\vec{r})=\epsilon_{i} \varphi_{i}(\vec{r}) .
$$

These are the Kohn-Sham equations [62], $\epsilon_{i}$ is the orbital energy of the corresponding Kohn-Sham orbital $\varphi_{i}$, and the density for this N-particle system is

$$
n_{s}(\vec{r})=\sum_{i}^{N}\left|\varphi_{i}(\vec{r})\right|^{2}
$$

The effective potential $v_{s}(\vec{r})$ will be chosen such that the ground state density of the auxiliary system $n_{s}(\vec{r})$ is equal to the original ground state density $n(\vec{r})$.

The key point of Kohn-Sham theory is to calculate the kinetic energy under the assumption of non-interacting electrons

$$
T_{s}[n]=T_{s}\left[\left\{\varphi_{i}[n]\right\}\right]=\sum_{i}\left\langle\varphi_{i}\left|-\frac{1}{2} \nabla^{2}\right| \varphi_{i}\right\rangle
$$

where the notation implies that $T$ depends on the $\left\{\varphi_{i}\right\}$ which in turn depends on the ground state density $n$. In reality, the electrons are interacting, and the (3.61) does not provide the total kinetic energy, but usually the most of it. The remaining kinetic energy is absorbed into an exchange-correlation term, and a general energy expression can be written as

$$
E[n]=T_{s}[n]+V_{e x t}[n]+J[n]+E_{x c}[n] .
$$

Now, equating this to the exact energy (3.57), we obtain a definition for $E_{x c}$

$$
E_{x c}[n]=\left(T[n]-T_{s}[n]\right)+\left(V_{e e}[n]-J[n]\right)
$$

i.e. the kinetic correlation energy plus the potential correlation and exchange energy. In this orbitals setting one can also think to write the exchange part following $\mathrm{HF}$ theory as

$$
E_{x}[n]=-\frac{e^{2}}{2} \sum_{i j} \iint \frac{\varphi_{i}^{*}\left(\vec{r}_{1}\right) \varphi_{j}^{*}\left(\vec{r}_{2}\right) \varphi_{i}\left(\vec{r}_{2}\right) \varphi_{j}\left(\vec{r}_{1}\right)}{\left|\vec{r}_{1}-\vec{r}_{2}\right|} d \vec{r}_{1} d \vec{r}_{2}
$$

leaving us with the main problem being correlation.

For the second HK theorem, the solution is the minimum of the functional (3.62), but since $T_{s}$ is now written as dependent from the effective orbitals, a direct minimization is not possible. In the Kohn-Sham scheme we can write for the interacting system

$$
\begin{aligned}
0=\frac{\delta E[n]}{\delta n(\vec{r})}=\frac{\delta T_{s}[n]}{\delta n(\vec{r})} & +\frac{\delta V_{e x t}[n]}{\delta n(\vec{r})}+\frac{\delta E_{H}[n]}{\delta n(\vec{r})}+\frac{\delta E_{x c}[n]}{\delta n(\vec{r})}= \\
& =\frac{\delta T_{s}[n]}{\delta n(\vec{r})}+v(\vec{r})+v_{H}(\vec{r})+v_{x c}(\vec{r})
\end{aligned}
$$


where $v(\vec{r})$ is the external potential, while $v_{H}(\vec{r})$ and $v_{x c}(\vec{r})$ are the functional derivatives of Hartree and exchange-correlation energy, respectively. On the other hand for the non-interacting Kohn-Sham system the minimization condition is simply

$$
0=\frac{\delta E_{s}[n]}{\delta n(\vec{r})}=\frac{\delta T_{s}[n]}{\delta n(\vec{r})}+\frac{\delta V_{s}[n]}{\delta n(\vec{r})}=\frac{\delta T_{s}[n]}{\delta n(\vec{r})}+v_{s}(\vec{r}),
$$

and the density solving this Euler-Lagrange equation is $n_{s}(\vec{r})$. Comparing equations (3.65) and (3.66) we find that both minimizations lead to the same solution $n(\vec{r})=n_{s}(\vec{r})$ if

$$
v_{s}(\vec{r})=v(\vec{r})+v_{H}(\vec{r})+v_{x c}(\vec{r}) .
$$

Now most of the energy is known and only the xc part has to be approximated with some functional. The xc potential

$$
v_{x c}[n](\vec{r})=\frac{\delta E_{x c}[n]}{\delta n(\vec{r})}
$$

can only be calculated explicitly once an approximation for $E_{x c}[n]$ has been chosen and since it depends on $n(\vec{r})$, which is unknown, the solution to KS equations has to be found self consistently. The flowchart in fig. 3.2 represents this SCF procedure.

The improvements brought by KS theory come at the price of reintroducing the molecular orbitals ( $3 \mathrm{~N}$ variables), analogous to the Hartree-Fock theory, but in a method that is still quite simple for being capable to capture many-particle correlation (once the right xc functional has been chosen). 


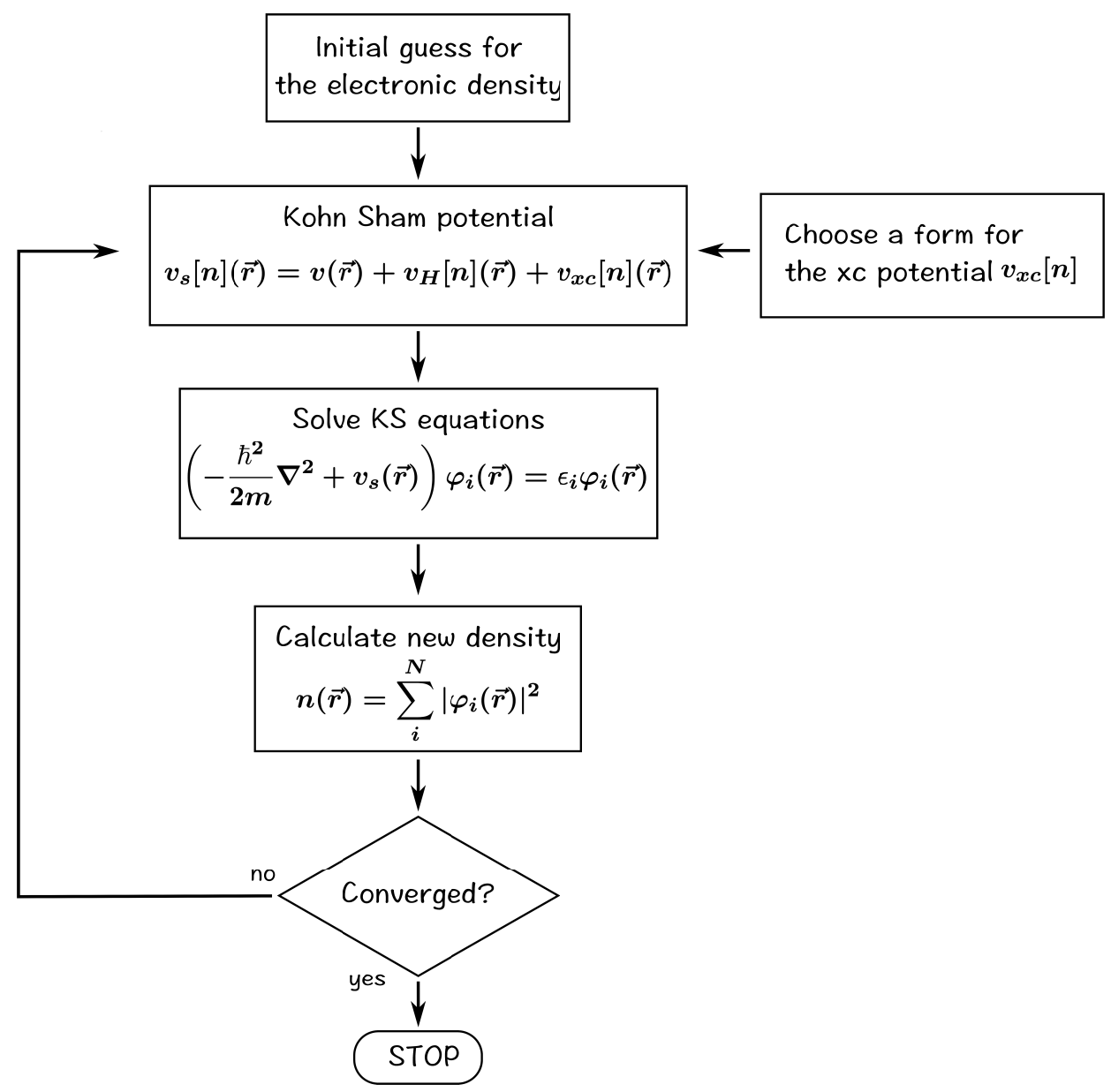

Figure 3.2. The SCF procedure for the Kohn-Sham scheme. 


\section{CHAPTER 4}

\section{Charge Transport in Organic Materials}

The charge transport is a complex problem that can be tackled in multiple different ways depending on the approximations we are willing to make. In an organic solar cell, when an exciton dissociate at the interface (chapter 1), the electron and the hole generated can occupy in general excited states on different molecules (hot CT state $[19,20,63])$. The charge transfer is thus in general involving quantum mechanical excitations of higher energy levels, fact that for simplicity is usually neglected in the simulations.

Furthermore, organic materials are soft, every molecule is bounded to another through van der Walls forces. When an extra electron is added in these materials, significant deformations (and consequently changes in nuclear vibrations) are happening on the charged molecule and on the nearby ones. When the electron is moving in the material these deformations will follow creating an effective quasiparticle, the polaron. If the vibrations are or not interpreted as phonons depends on the context.

When dealing with crystalline materials, usually a Hamiltonian picture including phonons is employed. The Hamiltonians of the electrons and the lattice are interacting and cannot be considered separately. This requires a coupled Hamiltonian (Holstein or Holstein Peierls) whose solution is non-trivial [29-31].

In amorphous materials the lack of crystallinity make the concept of phonons quite vague. The solution to this kind of problem is not possible analytically, the disorder of the material render the mathematical description very complex. Monte Carlo simulations (chapter 5) are usually employed to study transport in these materials. This reduces the problem to finding reasonable approximations for the rates of the charge hopping from one molecule to another. A popular method widely employed in the literature is Marcus theory [64]. In this case the vibrational degrees of freedom of every molecule are treated classically. More sophisticated methods like Levi-Jortner theory [65-67] allows to consider quantized vibrations 
and vibrational tunnel effects. An interesting debate is present in the literature on how much these nuclear tunnel effects are important for conduction in organic materials [68].

In this chapter we will present the main methods used in the literature to study charge transport in organic materials, following closely ref. [69] for section 4.1 and ref. [70] for sections 4.2 and 4.3 .

\subsection{Franck-Condon Principle}

A quantum system has some states $|\alpha\rangle$ and an Hamiltonian $H$. If we perturb the system with a time dependent perturbation $V e^{i \omega t}$ the transition probability per unit of time from a quantum state $|\alpha\rangle$ to another state $|\beta\rangle$ is given by the Fermi golden rule

$$
w_{\alpha \beta}=\frac{2 \pi}{\hbar}\left|V_{\beta \alpha}\right|^{2} \delta\left(E-E_{\beta \alpha}\right)
$$

where $E=\hbar \omega$ and we have assumed that $V$ is a "small" perturbation (in the sense of time-dependent perturbation theory). For electromagnetic radiation, $E$ is the energy of the single photons $(E=h \nu)$ and the first order approximation for the transition is:

$$
V_{\alpha \beta} \simeq\langle\alpha|\vec{D} \cdot \vec{E}| \beta\rangle .
$$

with $\vec{E}$ being the electric field and $\vec{D}$ the dipole of the system. For a molecule

$$
\vec{D}=e \sum_{i} Z_{i} \vec{R}_{i}-e \sum_{j} \vec{r}_{j}
$$

and the wave function $|\alpha\rangle$ can be decomposed, according to Born-Oppenheimer approximation, in an electronic and a nuclear vibrational-rotational part, with quantum numbers $n_{\alpha}, v_{\alpha}$ and $r_{\alpha}$ respectively,

$$
|\alpha\rangle=|\psi\rangle \otimes|\phi\rangle=\left|\psi_{n_{\alpha}}\right\rangle \otimes\left|\phi_{v_{\alpha}}\right\rangle \otimes\left|\phi_{r_{\alpha}}\right\rangle
$$

Identifying with $n_{\beta}, v_{\beta}$ and $r_{\beta}$ the quantum numbers of $|\beta\rangle$, the matrix elements for the electric dipole are

$$
\langle\beta|\vec{D}| \alpha\rangle=\left\langle\phi_{v_{\beta}} \phi_{r_{\beta}}\left|\left(\left\langle\psi_{n_{\beta}}\left|e \sum_{i} Z_{i} \vec{R}_{i}\right| \psi_{n_{\alpha}}\right\rangle-\left\langle\psi_{n_{\beta}}\left|e \sum_{j} \vec{r}_{j}\right| \psi_{n_{\alpha}}\right\rangle\right)\right| \phi_{v_{\alpha}} \phi_{r_{\alpha}}\right\rangle .
$$

The first term in (4.5) is zero when the electronic state is changing, because the operator does not depend on the electron coordinates and the two electronic wave functions are orthogonal $\left(\left\langle\psi_{n_{\beta}} \mid \psi_{n_{\alpha}}\right\rangle=\delta_{n_{\alpha} n_{\beta}}\right)$. The second term instead is the electronic dipole, parametrically dependent on the nuclear coordinates $\underline{\mathrm{R}}$, and the (4.5) becomes

$$
\langle\beta|\vec{D}| \alpha\rangle=\left\langle\phi_{v_{\beta}} \phi_{r_{\beta}}\left|\vec{D}_{e l}(\underline{\mathrm{R}})\right| \phi_{v_{\alpha}} \phi_{r_{\alpha}}\right\rangle .
$$


The dipole $\vec{D}_{e l}(\underline{\mathrm{R}})$ depends on the internal vibrational coordinates $\underline{\mathrm{q}}$ and, once calculated, the direction depends on the global rotation of the molecule $\theta, \varphi$

$$
\vec{D}_{e l}(\underline{\mathrm{R}})=\operatorname{Rot}(\theta, \varphi) \vec{D}_{e l}(\underline{\mathrm{q}}) .
$$

The Franck-Condon approximation consists in taking

$$
\vec{D}_{e l}(\underline{\mathrm{R}}) \simeq \vec{D}_{e l}\left(\underline{\mathrm{R}}_{e q}\right)=\operatorname{Rot}(\theta, \varphi) \vec{D}_{e l}\left(\underline{\mathrm{q}}_{e q}\right)=D_{e l}\left(\underline{\mathrm{q}}_{e q}\right) \hat{v}\left(\theta+\theta_{0}, \varphi+\varphi_{0}\right),
$$

i.e. the norm of the molecular dipole is calculated in the equilibrium position $\underline{q}_{e q}$ of all the vibrational coordinates when the system is in the initial electronic level $|\alpha\rangle$. $\hat{v}\left(\theta+\theta_{0}, \varphi+\varphi_{0}\right)$ is a versor pointing in the direction $\left(\theta+\theta_{0}, \varphi+\varphi_{0}\right)$, where $\left(\theta_{0}, \varphi_{0}\right)$ is the direction of $\vec{D}_{e l}\left(\underline{q}_{e q}\right)$. This famous approximations relies on the fact that the ground vibrational levels (from which usually excitations are calculated) are strongly localized near the equilibrium in the vibrational coordinates space. For this reason we can say that integrating in the vibrational coordinates $\left\langle\phi_{v_{\beta}}\left|\vec{D}_{e l}(\underline{\mathrm{R}})\right| \phi_{v_{\alpha}}\right\rangle$ the integrand function is different from zero only when the two vibrational wave functions overlap. Going back to equation (4.6), we can first integrate over the vibrational coordinates and achieve

$$
\langle\alpha|\vec{D} \cdot \vec{E}| \beta\rangle=f_{v_{\alpha} v_{\beta}}\left\langle J_{\beta}, M_{\beta}\left|\vec{D}_{e l}\left(\underline{\mathrm{R}}_{e q}\right)\right| J_{\alpha}, M_{\alpha}\right\rangle
$$

where we have made explicit the form of the rotational wave functions. The overlap integral $f_{v_{\alpha} v_{\beta}}$, between the two vibrational wave functions of the different electronic states, is also usually referred to as the Franck-Condon factor. It results that the ground state tends to be localized near the equilibrium point, while the higher vibrational states tend to be localized near the classical limit point. Thus a big result of Franck-Condon principle is stating that the most probable transitions are the vertical ones. This is compatible with the intuitive argument that the nuclei movements are slow compared to the electronic ones and that electronic transitions happen at still nuclei.

\subsection{Vibrational coupling on a single molecule}

Let us analyze the case of an isolated molecule, with internal vibrational coordinates $\underline{q}$ (displacements or rotations). Every vibrational coordinate $q$ contributes to the total Hamiltonian of the system with

$$
H_{n}^{v i b}=\frac{P^{2}}{2 M_{e f f}}+V_{n}(q),
$$

in which the potential function $V_{n}(q)$ depends on the electronic level $n$. For small elongations from the equilibrium position $q_{n}$, the vibrational potential can be approximated with an harmonic potential

$$
V_{n}(q) \simeq \frac{1}{2} M_{e f f} \omega_{n}^{2}\left(q-q_{n}\right)^{2}+v_{n}
$$


with effective mass $M_{\text {eff }}$, a characteristic angular frequency $\omega_{n}$ and an energy offset $v_{n} . V_{n}(q)$ is the potential felt by the nuclei in the situation in which only one out of the $N$ electrons of the system is excited to level $n$. We can argue and then also verify experimentally in some cases [70], that the curvature of $V_{n}(q)$ differs not strongly from that of the ground state potential $V_{0}(q)$. Hence, we can make the further approximation that the vibrational energy spacing $\hbar \omega$ is very similar for both potentials and we can use the same $\omega=\omega_{n}=\omega_{0}$ for every electronic level of a particular vibrational mode $q$.

Let us introduce the dimensionless nuclear coordinate

$$
\xi=\sqrt{\frac{M_{e f f} \omega}{2 \hbar}}\left(q-q_{0}\right),
$$

centered around the equilibrium position $q_{0}$ of the electronic ground state $(n=0)$. The first two vibrational potential are the ones involved in the first electronic transition, accordingly to previous approximations we can write

$$
\begin{aligned}
& V_{0}(\xi)=\hbar \omega \xi^{2}+v_{0} \\
& V_{1}(\xi)=\hbar \omega(\xi-g)^{2}+v_{1} .
\end{aligned}
$$

The exciton-phonon coupling constant $g$, introduced above, is the displacement of the excited state potential $V_{1}$ with respect to the ground state potential $V_{0}$ along the dimensionless coordinate $\xi$ (fig. 4.1). When a HOMO electron is excited, from the bottom of $V_{0}$, the molecule feels a sudden change of the nuclear potential (Franck-Condon). The nuclear coordinate $\xi$ remains momentarily approximately 0 as it was before. Introducing the vertical excitation energy for the molecule

$$
E_{\text {vert }}=V_{1}(0)-V_{0}(0)
$$

the excited state potential can be rewritten as

$$
V_{1}(\xi)=\hbar \omega(\xi-g)^{2}+v_{0}+E_{\text {vert }}-E_{\text {reorg }},
$$

where $E_{\text {reorg }}$ is the vibrational reorganization energy. This quantity gives the energy gain from the geometry relaxation into the new equilibrium geometry of the electronically excited molecule. Considering classical energy levels, thus forgetting the harmonic oscillator quantization and the zero point energy, we can connect $E_{\text {reorg }}$ with the exciton-phonon coupling constant by

$$
E_{\text {reorg }} \simeq \hbar \omega g^{2} \text {. }
$$

Remembering now the definitions of creation and destruction operators for the harmonic oscillator

$$
\begin{aligned}
b^{\dagger} & =\xi-\frac{1}{2} \frac{\partial}{\partial \xi} \\
b & =\xi+\frac{1}{2} \frac{\partial}{\partial \xi},
\end{aligned}
$$




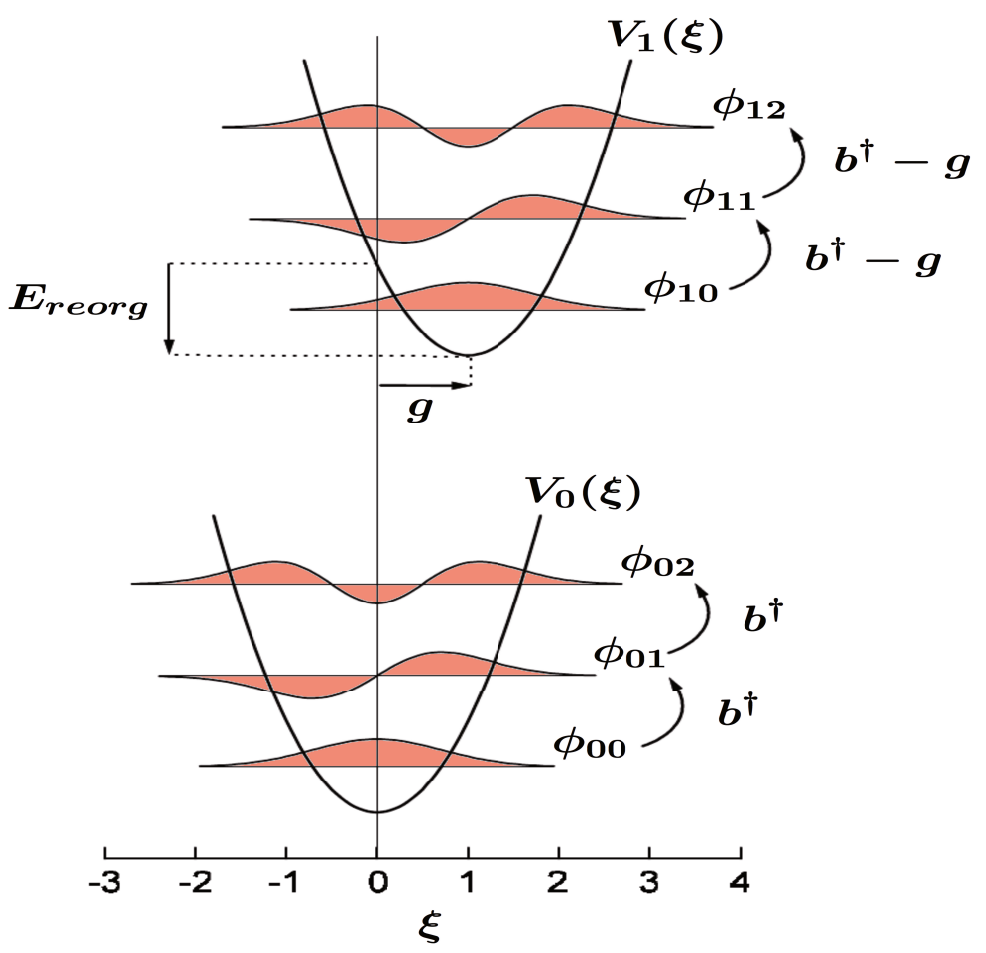

Figure 4.1. Translation of the harmonic potential due to electronic excitation. Figure adapted from [70].

the vibrational Hamiltonian in the electron ground state becomes

$$
H_{0}^{v i b}=\hbar \omega\left(b^{\dagger} b+\frac{1}{2}\right)+v_{0} .
$$

For the translated harmonic potential (in the excited case) the operators are expressed in terms of the translated coordinate $\tilde{\xi}=\xi-g$, resulting in

$$
\begin{aligned}
\tilde{b}^{\dagger} & =\tilde{\xi}-\frac{1}{2} \frac{\partial}{\partial \tilde{\xi}}=b^{\dagger}-g \\
\tilde{b} & =\tilde{\xi}+\frac{1}{2} \frac{\partial}{\partial \tilde{\xi}}=b-g
\end{aligned}
$$

and the Hamiltonian is

$$
\begin{aligned}
H_{1}^{v i b} & =\hbar \omega\left(\tilde{b}^{\dagger} \tilde{b}+\frac{1}{2}\right)+v_{0}+E_{\text {vert }}-E_{\text {reorg }}= \\
& =\hbar \omega\left(b^{\dagger} b+\frac{1}{2}\right)-\hbar \omega g\left(b^{\dagger}+b\right)+v_{0}+E_{\text {vert }}
\end{aligned}
$$


At this point, it is possible to combine the vibrational Hamiltonians for the ground and the first excited state by introducing electronic operators. Let us consider the two-level problem given by the electronic ground state $\left|\phi_{0}\right\rangle$ (HOMO) and the excited state $\left|\phi_{1}\right\rangle$ (LUMO). We use the electronic operators in the excited state $a^{\dagger}$ and $a$, which follows the rules

$$
\begin{array}{rlrl}
a^{\dagger}\left|\psi_{0}\right\rangle & =\left|\psi_{1}\right\rangle & a\left|\psi_{0}\right\rangle & =0 \\
a^{\dagger}\left|\psi_{1}\right\rangle & =0 & a\left|\psi_{1}\right\rangle & =\left|\psi_{0}\right\rangle .
\end{array}
$$

That means, $a^{\dagger}$ creates an exciton at the molecule, $a$ destroys it and $a^{\dagger} a$ is the exciton number operator (represents the presence of an excited electron on the molecule). Using this formalism, both operators $H_{0}^{v i b}$ and $H_{1}^{v i b}$ can be combined by multiplying the additional terms in $H_{1}^{v i b}$ with $a^{\dagger} a$. We obtain the monomer Hamiltonian

$$
H=\hbar \omega\left(b^{\dagger} b+\frac{1}{2}\right)+v_{0}+\left(-\hbar \omega g\left(b^{\dagger}+b\right)+E_{v e r t}\right) a^{\dagger} a
$$

where the exciton-phonon coupling $g$, resulting as explained before from the displacement of the excited vibrational potential. For organic conduction is interesting to have also the Hamiltonian for electron and hole transport. The derivation proceed exactly in the same way, considering the parameter $g$ as the electronphonon coupling or the hole-phonon coupling, given by the displacement of the vibrational potential due to the presence on the molecule of an extra electron or hole, respectively. The operators $a^{\dagger}$ and $a$ now have to be intended as the creation and destruction operators of an electron in the LUMO level or of a hole in the HOMO level.

\subsection{Holstein and Holstein-Peierls Hamiltonian}

The vibrational Hamiltonian for a crystalline structure is constructed as a composition of organic monomers Hamiltonian. Considering at first the monomers uncoupled, we simply add all the contributions of the form (4.23) coming from every lattice site $M$

$$
H=\sum_{M} \hbar \omega\left(b_{M}^{\dagger} b_{M}+\frac{1}{2}\right)+v_{0}+\left(-\hbar \omega g\left(b_{M}^{\dagger}+b_{M}\right)+E_{v e r t}\right) a_{M}^{\dagger} a_{M} .
$$

The zero vibrational energy

$$
\sum_{M}\left(\frac{\hbar \omega}{2}+v_{0}\right)
$$

in equation (4.24) can be set to a constant and so eliminated (translating the zero of potential energy). Joining the vibrational Hamiltonian (4.24) with the hopping part of Hubbard model [71], we obtain a crystalline Hamiltonian in which only 
local potential deformations are considered (an electron at site $M$ is coupled only with vibrations of the molecule situated at the same site $M$ )

$$
H=\sum_{M, N} \epsilon_{M N} a_{M}^{\dagger} a_{N}+\hbar \omega \sum_{M} b_{M}^{\dagger} b_{M}-g \hbar \omega \sum_{M}\left(b_{M}^{\dagger}+b_{M}\right) a_{M}^{\dagger} a_{M} .
$$

The term with $E_{\text {vert }}$ in (4.24) can be absorbed into the occupation energy $\epsilon_{M M}$ (thus including the vibrational energy needed for the excitation). The (4.25) is commonly referred to as the Holstein Hamiltonian, because of two pioneering works by Holstein on polarons $[72,73]$ and it is one of the simplest model systems for electron-phonon coupling [70].

Usually we deal with molecules, that possess several vibrational degrees of freedom $d$. This lead to the inclusion of just as many harmonic oscillators and each of these harmonic oscillator will be shifted by an electronic excitation. We then obtain $d$ coupling terms $\left\{g^{\eta}\right\}$, coupling the presence of the excitation $a^{\dagger} a$ to the creation and destruction operators, $\left\{b_{\eta}^{\dagger}\right\}$ and $\left\{b_{\eta}\right\}$ for the particular vibrational degree $\eta$ (with $\eta=1,2, \ldots, d)$. Applying the previous reasoning to every site of the lattice $M$, we obtain

$$
H=\sum_{M, N} \epsilon_{M N} a_{M}^{\dagger} a_{N}+\hbar \omega \sum_{M, \eta} b_{\eta, M}^{\dagger} b_{\eta, M}-\sum_{M, \eta} g^{\eta} \hbar \omega_{\eta}\left(b_{\eta, M}^{\dagger}+b_{\eta, M}\right) a_{M}^{\dagger} a_{M} .
$$

Let us assume $\mathcal{R}=\left\{\vec{R}_{M}\right\}$ are the set of lattice vectors (Bravais lattice). Transforming operators in momentum space through Discrete Fourier Transform it is possible to pass from localized operators to propagating operators [71]. Performing this transformation for the operators of the local vibrations $b$ and $b^{\dagger}$, we obtain the phonon operators

$$
\begin{aligned}
& b_{\mathbf{Q}}^{\dagger}=\frac{1}{\sqrt{N_{\mathcal{R}}}} \sum_{\vec{R} \in \mathcal{R}} b_{\eta, \vec{R}}^{\dagger} e^{i \vec{q} \cdot \vec{R}} \\
& b_{\mathbf{Q}}=\frac{1}{\sqrt{N_{\mathcal{R}}}} \sum_{\vec{R} \in \mathcal{R}} b_{\eta, \vec{R}} e^{-i \vec{q} \cdot \vec{R}},
\end{aligned}
$$

where the couple $\mathbf{Q}=(\eta, \vec{q})=$ (branch index, phonon momentum) individuates a phonon state and $N_{\mathcal{R}}$ is the number of points of the lattice. Substituting for these operators in the Holstein Hamiltonian and allowing for an hopping electron-phonon coupling (through a tensor of coupling constants $g_{M N}^{\mathbf{Q}}$ for every phonon $\mathbf{Q}$ ) we obtain the Holstein-Peierls Hamiltonian

$$
\begin{aligned}
H=\sum_{M, N} \epsilon_{M N} a_{M}^{\dagger} a_{N}+\sum_{\mathbf{Q}} \hbar \omega_{\mathbf{Q}} b_{\mathbf{Q}}^{\dagger} b_{\mathbf{Q}}+ & \\
& +\sum_{M N} \sum_{\mathbf{Q}} \hbar \omega_{\mathbf{Q}} g_{M N}^{\mathbf{Q}}\left(b_{\mathbf{Q}}^{\dagger}+b_{-\mathbf{Q}}\right) a_{M}^{\dagger} a_{N} .
\end{aligned}
$$

The coupling constant $g_{M N}^{\mathrm{Q}}$ couples the hopping between localized electron states $N$ and $M$ to the propagating phonon $\mathbf{Q}$. 


\subsection{Marcus Formula}

Marcus Theory [64] was initially formulated to explain the rates of electron transfer reactions, i.e. those chemical reactions in which an electron moves from one chemical species (the electron donor) to another (the electron acceptor). It is widely used in the literature to calculate the charge carriers transfer rate between two molecules. Marcus Formula can be derived from Fermi golden rule and it expresses the rate of the hopping of an electron from the ground state of the initial molecule $i$ to the ground state of the final molecule $j$, moving always on the lowest of the two energy curves.

Let us consider a single molecule immersed in a continuous electric field $(\omega=0)$. In the Born-Oppenheimer approximation (4.4), every vibrational coordinate $q$ generates a manifold of vibrational states (usually approximated as states of an harmonic oscillator). The probability of an electronic transition from the ground electronic level $\left|\psi_{i}\right\rangle$ to an excited one $\left|\psi_{f}\right\rangle$ can be calculated through the Fermi golden rule summed over all the possible vibrational transitions

$$
w_{i f}=\sum_{\alpha \beta} w_{\alpha \beta}=\frac{2 \pi}{\hbar} \sum_{\alpha \beta} f\left(E_{\alpha}\right)\left|V_{\beta \alpha}\right|^{2} \delta\left(E_{\beta}-E_{\alpha}\right) .
$$

$f\left(E_{\alpha}\right)$ is the probability of being in the $|\alpha\rangle$ level and $V_{\beta \alpha}$ is the matrix element for the transition from $|\alpha\rangle$ to $|\beta\rangle$, where we have set $n_{\alpha}=i$ and $n_{\beta}=f$. Assuming to be in the "classical regime" for the occupation of the vibrational quantum levels, this probability is given by the Boltzmann factor

$$
f\left(E_{\alpha}\right)=\frac{e^{-\frac{E_{\alpha}}{k_{B} T}}}{\sum_{\alpha^{\prime}} e^{-\frac{E_{\alpha^{\prime}}}{k_{B} T}}} .
$$

As a further assumption, changing our vision of the harmonic oscillator from quantum to classical, we readjust the Boltzmann factor focusing now on the probability for the system of being in the position $q$. After normalization we can obtain the probability of being at $q$ as

$$
f(q)=\sqrt{\frac{M \omega}{2 \pi k_{B} T}} e^{-\frac{E(q)}{k_{B} T}}
$$

where $E(q)$ is the classical energy for the position $q$. According to Franck-Condon theory, the most probable transitions from the ground state of the harmonic oscillator are the vertical ones. Thus the Fermi golden rule become

$$
w_{i f}=\frac{2 \pi}{\hbar} \int d q f(q)\left|V_{i f}(q)\right|^{2} \delta\left(E_{f}(q)-E_{i}(q)\right),
$$

with $V_{i f}(q)$ the matrix element between the electronic levels $i$ and $f$ electron level assuming the value $q$ of the vibrational coordinate stays fixed (fixed nuclei). $V_{i f}(q)$ is commonly approximated by a single value $V_{i f}=V_{i f}\left(q_{e q}\right)$ and taken outside the integration sign. $q_{e q}$ can be the equilibrium value of the coordinate $q$ in the 
electronic level $i$ or some other kind of equilibrium value chosen for the problem at hand. Let us now develop the delta inside the integral (4.33). We know from distribution theory that

$$
\delta(f(x))=\sum_{i} \frac{\delta\left(x_{i}\right)}{\left|f^{\prime}\left(x_{i}\right)\right|}
$$

where the $x_{i}$ are the zeros of the function $f$. Approximating both the potential energy surfaces of the nuclei as parabolas with the same curvature, we obtain

$$
\begin{aligned}
E_{f}(q)-E_{i}(q) & =\frac{1}{2} M \omega^{2}\left(q-q_{f}\right)^{2}+E_{f 0}-\frac{1}{2} M \omega^{2}\left(q-q_{i}\right)^{2}-E_{i 0}= \\
& =\Delta E_{0}+\frac{1}{2} M \omega^{2}\left(q_{f}^{2}-q_{i}^{2}\right)-M \omega^{2} q\left(q_{f}-q_{i}\right) .
\end{aligned}
$$

This is a linear function in $q$ and the zero of this function $q^{*}$ is the point in which the two energies (of initial and final state) are equal

$$
q^{*}=\frac{\Delta E_{0}+\frac{1}{2} M \omega^{2}\left(q_{f}^{2}-q_{i}^{2}\right)}{M \omega^{2}\left(q_{f}-q_{i}\right)} .
$$

The delta of expression (4.33) becomes

$$
\delta\left(E_{f}(q)-E_{i}(q)\right)=\frac{\delta\left(q-q^{*}\right)}{M \omega^{2}\left|q_{f}-q_{i}\right|},
$$

that substituted in the (4.33) itself, gives

$$
w_{i f}=\frac{2 \pi}{\hbar}\left|V_{i f}\right|^{2} \sqrt{\frac{1}{4 \pi k_{B} T \lambda}} e^{-\frac{\Delta E^{*}}{k_{B} T}} .
$$

$\Delta E^{*}$ is the classical barrier to overcome in passing from initial to final state and $\lambda$ is the classical reorganization energy after a vertical excitation

$$
\begin{aligned}
\Delta E^{*} & =\frac{1}{2} M \omega^{2}\left(q^{*}-q_{i}\right)^{2} \\
\lambda & =\frac{1}{2} M \omega^{2}\left(q_{i}-q_{f}\right)^{2} .
\end{aligned}
$$

Substituting the (4.36) into (4.39) and considering definition (4.40), we get, after several passages

$$
\Delta E^{*}=\frac{\left(\Delta E_{0}+\lambda\right)^{2}}{4 \lambda}
$$

and substituting this expression in (4.38) we obtain the Marcus transfer rate expression

$$
w_{i f}=\frac{2 \pi}{\hbar}\left|V_{i f}\right|^{2} \sqrt{\frac{1}{4 \pi k_{B} T \lambda}} \exp \left(-\frac{\left(\Delta E_{0}+\lambda\right)^{2}}{4 \lambda k_{B} T}\right) .
$$




\section{CHAPTER 5}

\section{Monte Carlo Simulations}

In the 1930s, Fermi used sampling to estimate quantities involved in controlled fission [74]. This sampling was performed by hand or using a mechanical device called "Fermiac". At the time of the Manhattan project in Los Alamos, John von Neumann and Stanislaw Ulam used computers to generate the system samples, getting results in a relatively fast and automatic way. Being secret, the work of von Neumann and Ulam required a code name, the name Monte Carlo, refers to the Monte Carlo Casino in Monaco where Ulam's uncle would go to gamble borrowing money from relatives [75]. In 1949 Metropolis and Ulam published their results in the Journal of the American Statistical Association [76].

Monte Carlo methods are widely used in statistical simulations of physical systems. The core idea of Monte Carlo is to study a system using repeated random sampling to obtain numerical results. This approach is often the simplest way to solve a problem, and sometimes the only feasible way. The main drawback of Monte Carlo methods is that, depending on the system, they may require a very high number of steps to reach a reasonable convergence and, for this reason, they can be computationally expensive to carry out. Nevertheless, with the continuous improvement of modern computers, this problem is getting progressively less relevant over time.

An important and well studied type of Monte Carlo technique is the Markov Chain Monte Carlo method (MCMC) [77]. In this work we will use a particular version of MCMC, called Gibbs sampling. Let us consider a system of interest described by several random variables $A, B, C, \ldots$ and let us say we are interested in simulating the evolution of the system, sampling from the joint distribution $P(A, B, C, \ldots)$. This distribution is often complicated and difficult to calculate, if not impossible. The main idea of Gibbs sampling is to sample one variable at the time, while keeping the others fixed. At every step thus only one variable of the system is varied and the sequence obtained by such evolution is forming 
a Markov chain. It can be demonstrated that, under particular conditions and once the Markov chain has reached equilibrium, sampling from the Markov chain is equivalent as sampling from a probability distribution called the equilibrium distribution. Designing an MCMC properly, it is thus possible to sample from a particular probability distribution of interest. More details on Markov chains and MCMC algorithms can be found in [77].

\subsection{Kinetic Monte Carlo Algorithm}

Kinetic Monte Carlo algorithm (KMC) is a variant of Monte Carlo simulation intended to simulate the time evolution (hence the term kinetic) of a particular process of interest. In our charge transport context, as KMC we mean a particular variant of Gibbs sampler. We will explain in details this algorithm in the following of the present section.

A particular molecular orbital level in the system is specified with an upper case letter $M=(i, \alpha)$, identifying the orbital level $\alpha$ of the molecule $i$. We will imply in the notation that the orbital $M$ belongs to the molecule $i$ while the orbital $N$ belongs to the molecule $j$ (where $i$ and $j$ are different molecules). The flowchart of the algorithm is presented in figure 5.1. The initial condition (point 1) is composed by the MD simulated structure and by the initial position of the charges involved in the simulation. At a given time, every charge carrier hops from one molecular level $M$ to another molecular level $N$ with a rate given by the Marcus formula (section 4.4)

$$
w_{M N}=\frac{2 \pi}{\hbar}\left|H_{N M}\right|^{2} \sqrt{\frac{1}{4 \pi k_{B} T \lambda_{N M}}} \exp -\frac{\left(\Delta E_{N M}+\lambda_{N M}\right)^{2}}{4 \lambda_{N M} k_{B} T} .
$$

$H_{M N}$ is the transfer integral expressing the electronic coupling of the levels $M$ and $N, \Delta E_{N M}$ is the energy difference between their two ground vibrational states and $\lambda_{N M}$ is the reorganization energy required for all the structural adjustments of the system after the hopping has happened. For the charge carrier on the orbital $M$, we calculate the rates $w_{M N}$ for every possible acceptor orbital $N$ within a given cut-off (point 2). The total escape rate for the charge on the orbital $M$ is thus $R_{M}=\sum_{N} w_{M N}$. In the general case of multiple charge carriers, we can think of every charge carrier $a$ sitting on the orbital $M_{a}$ and having a total escape rate $R_{M_{a}}$ (point 3). The evolution of the system is determined thanks to a weighted random selection algorithm (WRS), where the probability of selecting a particular value depends on its associated weight. First the hopping charge carrier is selected using as weights the total escape rates $R_{M_{a}}$. This select the charge $c$ that is jumping, thus identifying the origin of the hop (point 4). Let us assume that the hopping charge $c$ is sitting on the orbital $M$. The particular hop to perform is selected with another WRS using as weights the $w_{M N}$. In this way we select the destination of the hop (point 5 ). The time $\Delta t$ after which this hop occurs is extracted from an exponential distribution $\sim R_{M} \exp \left(-R_{M} \Delta t\right)$, where $R_{M}$ is the total escape rate from the orbital $M, R_{M}=\sum_{N} w_{M N}$ (point 6). Now the hop is performed and the time is updated (point 7). Several stopping criteria can be specified. A stopping 


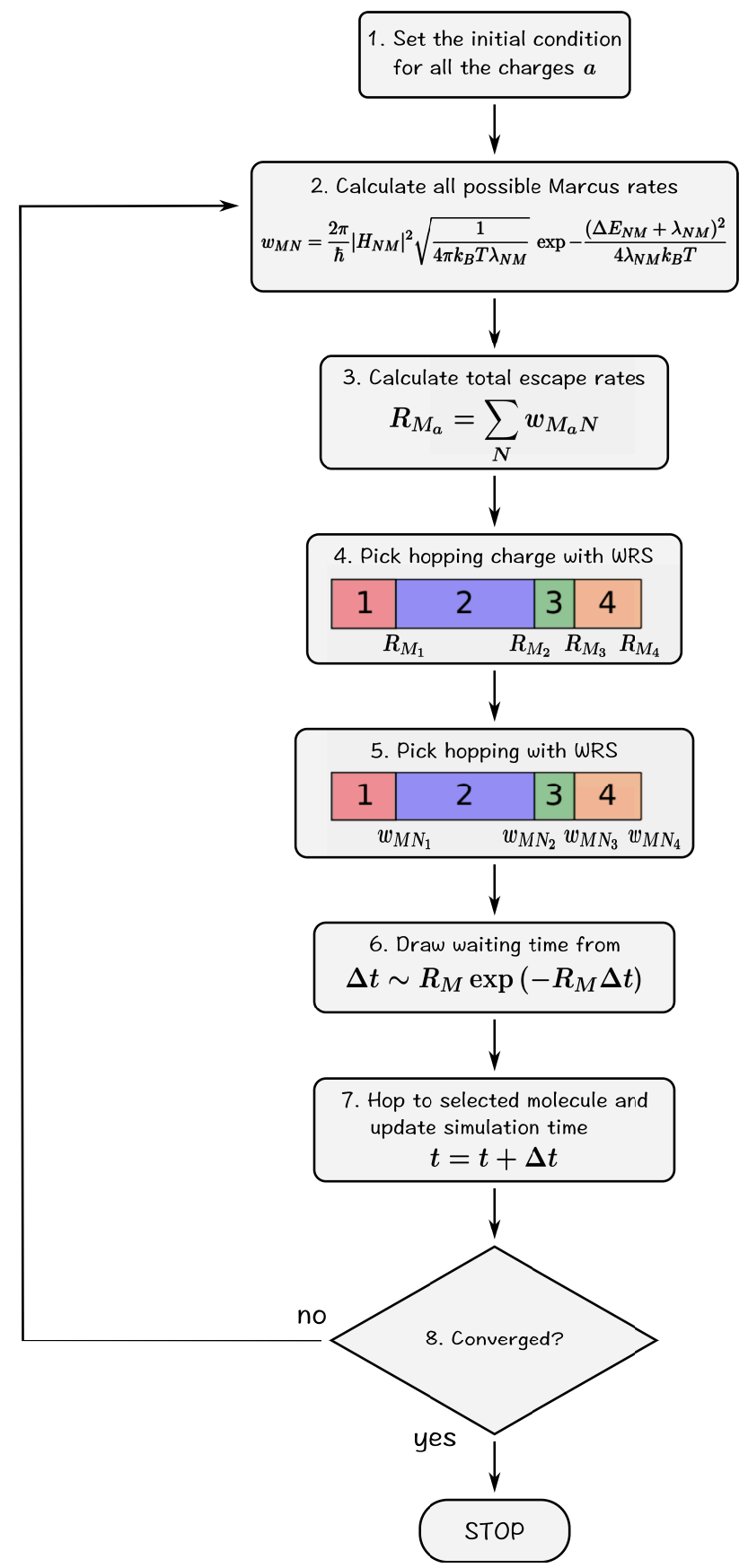

Figure 5.1. Flowchart of the KMC simulation used in this thesis. 
criterion can be the simulation time, the number of hops, but also some positional criteria like the charge carrier passing through a particular plane in space. If no stopping criterion is respected (point 8), we go back at point 2 and continue the simulation.

\subsection{Marcus Parameters Calculation}

For every possible hopping, we need to calculate three parameters: the energy difference $\Delta E$, the transfer integral $H_{N M}$ and the reorganization energy $\lambda$. The temperature $T$ and the external electric field $\vec{E}_{\text {ext }}$ are given as input of the simulation. $T$ is used directly in Marcus formula for the hopping rates, while $\vec{E}_{\text {ext }}$ is giving its contribution to the $\Delta E$. The modification that $\vec{E}_{\text {ext }}$ introduces on the transfer integrals $[78,79]$ represent a correction and will be completely neglected in our scheme. The effect of $\vec{E}_{\text {ext }}$ on the external reorganization energy has been discussed in Paper II. Since no crystallinity is assumed this scheme (fig. 5.1) translates in a huge amount of parameters even with the introduction of a distance cut-off. To calculate these parameters then, we have to make choices that allows us to deal with the computational complexity of the model. The calculation of every parameter is given in some detail in this section, further details can be found in Papers I and II.

\subsubsection{Site Energies}

The energy $\Delta E_{N M}=u_{N}-u_{M}$ is the difference between the energies of the two orbitals considered ( $M$ and $N)$. The single site energy $u_{M}$ is composed of several contributions that will be explained below:

$$
u_{M}=\epsilon_{M}+E_{M}^{p e r m-e l}(\underline{s})+E_{M}^{i n d-e l}(\underline{s})+E_{M}^{f i e l d}\left(s_{i}\right) .
$$

The first term $\epsilon_{M}$ in equation (5.2) is the orbital energy obtained from a QM calculation. Since these calculations are done for every molecule in the box, this value takes into account the energy shift caused by the conformational changes of the molecule in the bulk.

The other terms arise from the interaction of the charged molecule with its environment. They are dependent on the quantum state of the charged molecule, due to the extra charge on the orbital $M$. A cut-off is used for the Coulombic interactions, so that a charge located on the orbital $M$ interacts only with the molecules inside its interaction set $I(M)$, i.e. the set composed by the molecules nearer than the cut-off. For the polarization we use another cut-off, leading to a different interaction set denoted by $I_{p}(M)$, see fig. 5.2. The array $\underline{s}=$ $\left(s_{1}, s_{2}, \ldots, s_{i}, s_{j}, \ldots\right)$ is composed by the quantum states of every molecule in the system. A molecule can change quantum state, so that $\underline{s}$ can vary over time as a consequence of a hopping process. For the cases considered in Paper I-III, a molecule can only be neutral $(n)$ or charged $(c)$, where charged means different things depending on the molecule considered. For D molecules charged means one extra hole in the HOMO level, while for A molecules means on extra electron 


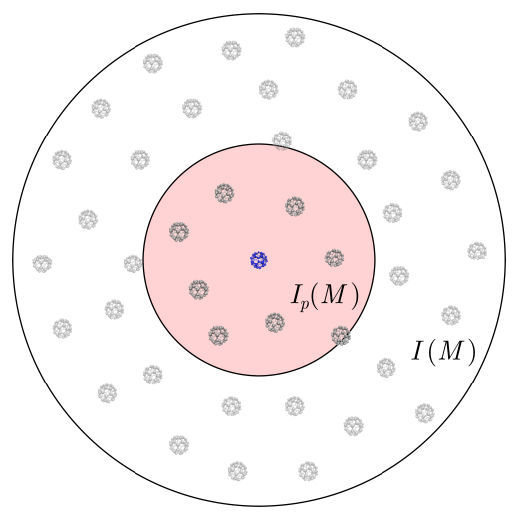

Figure 5.2. The interaction sets used for Coulombic interactions $I(M)$ and polarization $I_{p}(M)$, for a charge carrier situated on the molecular orbital $M$.

in the LUMO level. The atomic charges and polarizabilities of a molecule will change according to the molecular quantum state during the simulation. In the $\mathrm{C}_{60}$ example, when an electron moves from the molecular level $M=(i, L U M O)$ to the molecular level $N=(j, L U M O)$, the system passes from $s_{1}=(n, n, \ldots, c, n, \ldots)$ to $\underline{s_{2}}=(n, n, \ldots, n, c, \ldots)$ (fig. 5.3 on the top).
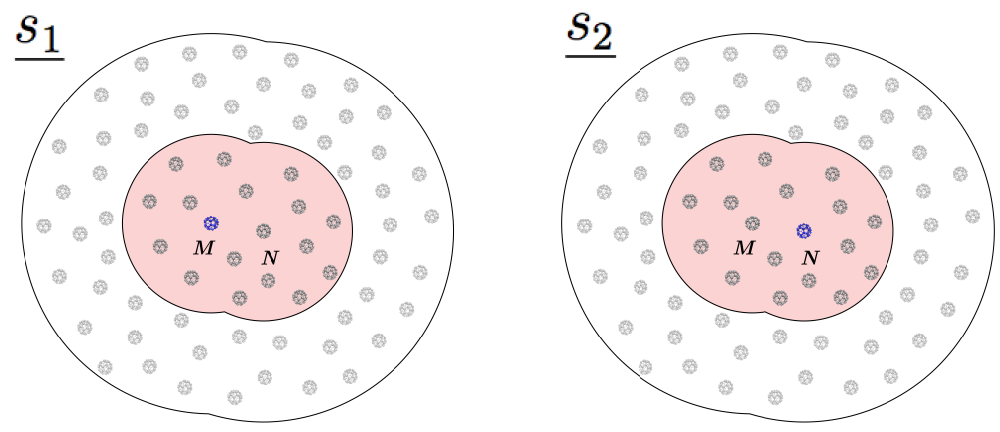

Figure 5.3. Illustration of two quantum states $\underline{s_{1}}$ and $\underline{s_{2}}$ whose difference is a charge hopping.

The second term in equation (5.2) represents the permanent electrostatic energy

$$
E_{M}^{p e r m-e l}(\underline{s})=\frac{1}{4 \pi \epsilon_{0}} \sum_{a \in i} \sum_{\substack{b \in h \\ h \in I(M)}} \frac{q_{a}\left(s_{i}\right) q_{b}\left(s_{h}\right)}{\left|\overrightarrow{r_{a}}-\overrightarrow{r_{b}}\right|}
$$

where $h$ is a molecule belonging to the interaction set $I(M)$ and $a, b$ are atoms. This sum is based on the atomic charges and thus includes the contribution of molecular permanent dipoles, quadrupoles, etc. The atomic charge $q_{a}$ on the atom 
$a$ depends on the quantum state of molecule $i$, which the atom belongs to. This is why it has been denoted as $q_{a}\left(s_{i}\right)$.

The third term of (5.2) is the energy due to polarization. Polarization in an organic material arises from two different contributions. The first contribution is given by the reorganization of the permanent multipoles of nearby molecules through the movement of their nuclei. This process is slow compared to the timescale of a charge carrier hopping and will be neglected in our model. The second contribution is faster and comes from the polarization of the nearby electronic clouds. This can be accounted for through atomic polarizabilities and induced dipoles. The resulting polarization energy is

$$
E_{M}^{i n d-e l}(\underline{s})=\frac{1}{4 \pi \epsilon_{0}} \sum_{a \in i} \sum_{\substack{b \in h \\ h \in I_{p}(M)}} \frac{q_{a}\left(s_{i}\right) \overrightarrow{p_{b}}(\underline{s}) \cdot\left(\overrightarrow{r_{a}}-\overrightarrow{r_{b}}\right)}{\left|\overrightarrow{r_{a}}-\overrightarrow{r_{b}}\right|^{3}},
$$

where $p_{b}(\underline{s})$ represents the induced dipole at the atom $b$, given that the nearby molecules are in the state $\underline{s}$. Since we want to avoid border effects, the interaction set $I_{p}(M)$ chosen for the polarization is always smaller than the one chosen for the permanent charges $I(M)$. The dipoles inside $I_{p}(M)$ are calculated depending on the permanent charges of all the molecules in the interaction set $I(M)$.

Finally, the last term of equation (5.2) consider the field contribution to the site energies given by

$$
E_{M}^{\text {field }}\left(s_{i}\right)=-\vec{E}_{e x t} \cdot \sum_{a \in i} q_{a}\left(s_{i}\right) \overrightarrow{r_{a}}
$$

where $\vec{E}_{\text {ext }}$ is the external field considered.

We use a nomenclature specifying the energy assignment method that has been used. ' $\mathrm{O}$ ' is for the $\mathrm{QM}$ orbital energies, ' $\mathrm{C}$ ' for the Coulombic interactions (5.3) and ' $\mathrm{P}$ ' for the polarization energies (5.4). $\mathrm{O}$ is used in case the energy is composed only by the QM orbital energies, OC when the energy is composed by the orbitals corrected by Coulombic interactions and OCP when all the three contributions are included.

\subsubsection{Transfer Integrals}

Transfer integrals can be calculated in different ways. One of the simplest method to calculate the transfer integral is called the energy-splitting-dimer (ESD) method $[78,80]$. This method is commonly used for organic semiconductors. When the extra charge is equally distributed over the two molecules, the energy difference between $M$ and $N$ is approximately equal to $2 H_{M N}$. Therefore, if the geometries of the molecules are known, the transfer integral can be calculated. To simplify the calculations, the geometries are often approximated as geometries of a neutral dimer.

Another way to calculate the transfer integral is through a weighted Mulliken formula [81] for carbon atoms [82]. This approach has been used in ref. [39] and it is based on the approximation that only p-electrons contributes to the transfer 
integral. It provides a quick and approximated formula but still gives a dependence of the transfer integrals on the relative orientation.

If wishing for a better description, we can turn to QM calculations. For every pair of nearby molecules (nearer than a chosen cut-off), three QM calculations are performed: one for the pair and two for the single molecules. The Fock matrix of the pair is then projected on the single molecular orbitals basis (the basis of the molecular orbitals of the two single molecules) and the transfer integrals $\left|H_{N M}\right|$ can be extracted as the non-diagonal elements of this rotated Fock matrix in the single molecular orbitals basis. This is known in literature as the projection method. It was originally formulated for ZINDO [83] and it has been subsequently extended [84] for more sophisticated QM calculations that do not assume orthogonality of atomic orbitals.

\subsubsection{Reorganization Energies}

The reorganization energy $\lambda$ is the energy required for all the structural adjustments of the system, after the charge hopping has occurred. It can be decomposed in two parts, an internal and an external reorganization contribution

$$
\lambda=\lambda_{\text {int }}+\lambda_{\text {out }} .
$$

The internal reorganization energy $\left(\lambda_{i n t}\right)$ is given by the internal geometry rearrangement of the molecule, after a charge is added or subtracted to the molecule. Let us consider a charge hopping from molecule $i$ to molecule $j$. The internal reorganization energy is the sum of the reorganization energy of the molecule $i$ that is passing from charged to neutral state and the reorganization energy of the molecule $j$ that is passing from neutral to charged state

$$
\lambda_{i n t}=\lambda_{i}^{c n}+\lambda_{j}^{n c} .
$$

These energies can be calculated performing quantum mechanical geometry reoptimizations on neutral and charged molecules and analyzing the energies obtained: $[38,85]$

$$
\begin{gathered}
\lambda_{i}^{c n}=E_{i}^{n C}-E_{i}^{n N} \\
\lambda_{j}^{n c}=E_{j}^{c N}-E_{j}^{c C}
\end{gathered}
$$

where for example $E_{i}^{n C}$ is the energy of molecule $i$ in the geometrical conformation of the charged molecule $(C)$, but in the neutral electronic configuration $(n)$. Since geometry optimizations at the quantum mechanical level are computationally expensive, these calculations are done once for all for a single isolated molecule and the resulting $\lambda_{\text {int }}$ is used for all the molecules in the system.

The external reorganization energy $\left(\lambda_{\text {out }}\right)$ is given by the re-polarization of the environment after the charge carrier's jump. As for the single site energies, we neglect the rearrangement of the permanent nearby multipoles, i.e. we do not perform molecular dynamics during the KMC simulation. This structural rearrangement is much slower than both the wave functions polarization and the internal reorganization of the molecule (fast reorganization of covalent bonds). 
The polarization contribution is taken into account by means of induced dipoles on the nearby molecules. Let us consider the energy contribution

$$
E_{M}^{i n d-e l}\left(\underline{s_{1}}, \underline{s_{2}}\right)=\frac{1}{4 \pi \epsilon_{0}} \sum_{a \in i} \sum_{\substack{b \in h \\ h \in I_{p}(M)}} \frac{q_{a}\left(s_{1 i}\right) \vec{p}_{b}\left(\underline{s_{2}}\right) \cdot\left(\overrightarrow{r_{a}}-\overrightarrow{r_{b}}\right)}{\left|\overrightarrow{r_{a}}-\overrightarrow{r_{b}}\right|^{3}}
$$

where as above $a$ and $b$ are atoms, orbital $M$ belongs to molecule $i$ and $h$ is a molecule in the polarization interaction set of $M$. This is the interaction energy between the charges on $i$ as given by the quantum state $s_{1}$ and the nearby dipoles induced by the quantum state $\underline{s_{2}}$. When a jump from $M \overline{\text { to }} N$ occurs, the quantum states are modified from $s_{1}$ to $s_{2}$, i.e. the state of $i$ changes from charged to uncharged and the state of $\bar{j}$ changes from uncharged to charged. An illustration of the states $\underline{s_{1}}$ and $\underline{s_{2}}$ with their respective induced dipoles are presented in fig. 5.4. The expression we are looking for has to describe the energy required for the
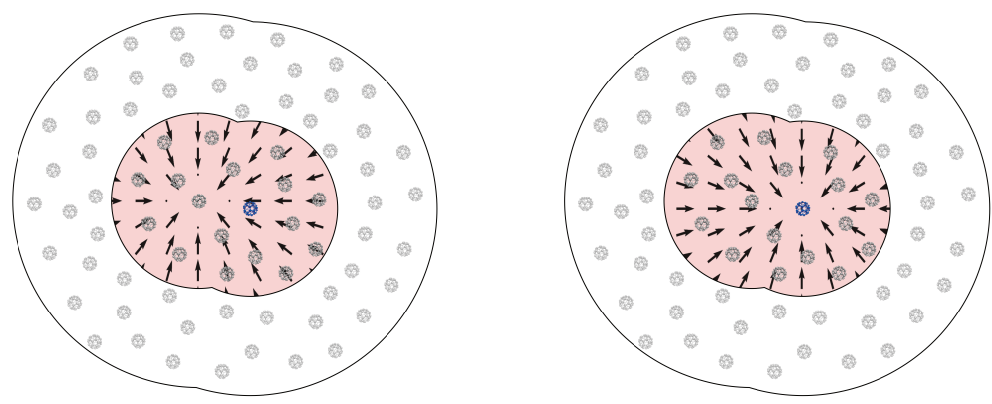

Figure 5.4. After the hop from $M$ to $N$, the situation before and after the reorganization.

external dipoles rearrangement, i.e. the difference between the energy of the new charge configuration in the old dipole arrangement (the jump happened but the structure did not relax yet) and the energy of the new charge configuration in the new dipole arrangement (system has re-polarized through dipoles reorganization). Consequently we can write the external reorganization energy as

$$
\lambda_{\text {out }}=E_{M}^{i n d-e l}\left(\underline{s_{2}}, \underline{s_{1}}\right)+E_{N}^{i n d-e l}\left(\underline{s_{2}}, \underline{s_{1}}\right)-E_{M}^{i n d-e l}\left(\underline{s_{2}}, \underline{s_{2}}\right)-E_{N}^{i n d-e l}\left(\underline{s_{2}}, \underline{s_{2}}\right) .
$$

The first and second terms are the energies given by the interaction of the atomic charges of molecules $i$ and $j$ in the state given by $\underline{s}_{2}$ (after the hop) with the induced dipoles given by the state $\underline{s}_{1}$ (before the hop). The remaining two terms are the energies of the interaction of the atomic charges of molecules $i$ and $j$ in the state given by $\underline{s}_{2}$ with the induced dipoles given by the same state $s_{2}$ (final situation after the hop). Note that for the reorganization energy we choose to neglect the energetic contribution between the electric field and the induced dipoles. We think this contribution is of secondary importance and we decided to neglect it (see more in Paper II). 


\section{CHAPTER 6}

\section{Summary and Results}

Kinetic Monte Carlo simulations have been performed with the algorithm presented in the previous chapter. In a continuous iterative process, the model has been progressively tested and refined till the present version. The main focus of the research papers produced is about mobility in organic materials and CT state splitting at the interface of an organic solar cell. The results and a brief description of the work will be presented in the following three sections.

\subsection{Energy correlation}

One of the first pioneering works on the simulations of charge transport in organic materials was published by Bässler [35]. These simulations were based on a crystalline lattice using a simple Miller-Abrahams hopping rate [86]. The disorder of the electronic levels (LUMO for electrons and HOMO for holes) of the system has been obtained by considering all the molecular energy levels independently and identically distributed (i.i.d.) random variables. The probability density of each of these random variables is a Gaussian distribution representing the DOS of the considered level. This approach allowed to gain great insight into the charge transport in organic materials and also to achieve qualitatively the correct mobility temperature dependence [35]. Also, the importance of considering a realistic spatial disorder in the bulk has been showed in the literature [39]. In this paper, the hole transport in Poly-Phenylenevinylene has been studied using chains of 4 and 6 monomers. Molecular dynamics (MD) simulations has been used to obtain the structure and the transfer integral calculated according to a weighted Mulliken formula [81] for carbon atoms [82]. In ref. [39], the temperature dependence of mobility is in agreement with experiments, but the field dependence is not. This problem in the simulations has been studied deeper and solved in Paper I. 
It is known that the functional dependence of the mobility on the field depends on the correlation of the energetic landscape [87]. Indeed using the correlation for randomly oriented dipoles (randomly oriented molecules in an amorphous material) derived by Novikov and Vannikov [88,89], the Poole-Frenkel formula [90] can be derived theoretically in what is called the correlation region [87]. Three regions of interest are identified in the literature for the mobility field dependence: the percolation region, the correlation region and the inverse Marcus region. The dipole correlation (or more in general the correlation given by atomic charges) is exactly the missing piece that is lost when the energy of the conducting levels in KMC simulations are sampled from i.i.d. Gaussian distributions. For this reason we computed the energy shift of the conducting level of every molecule in the box, allowing us to retrieve the expected Poole-Frenkel mobility. Also the transition fields separating the 3 regions of interest has been identified, allowing us to estimate the position of these regions on the field axis, see Paper I. This revealed to be a very useful tool in determining the interesting field strengths to consider for our simulations, used in Papers II and III.

\subsection{Polarization}

Another interesting benchmark molecule to test our scheme is represented by $\mathrm{C}_{60}$. This is a simple well studied material for which experimental mobility field dependence and mobility temperature dependence are available [91,92]. $\mathrm{C}_{60}$ is a spherical fullerene molecule, presenting no electrostatic permanent dipoles or multipoles in its neutral state. In this material then, it was not clear what is the effect producing the broadening of the DOS and the respective correlation of the energies, if there is any. When neglecting polarization of the environment, poor results are obtained in terms of both mobility field and temperature dependence. Polarization effects have been considered in Paper II as induced dipoles on the nearby molecules, leading to an energy correction for the single site energies and to an external reorganization happening after each hopping. In particular, the external reorganization energy revealed to be the most important factor, shifting the inverse Marcus region to higher fields and allowing a good qualitative agreement with experiments for both mobility field and temperature dependence.

\subsection{CT state splitting}

After having studied single charge carriers movement, we proceed to study the dynamics of multiple charges. In particular we focus on the CT state splitting (Paper III), that is intimately related to the efficient generation of free charge carriers in a solar cell (chapter 1 ). We build two kind of anthracene- $\mathrm{C}_{60}$ interfaces and perform KMC simulations at a range of applied electric fields, and with the fields applied at a range of angles with respect to the interface. The results show that depending on the relative orientation of the anthracene and $\mathrm{C}_{60}$ molecules, $\mathrm{CT}$ state splitting shows different behaviour with respect to both applied field strength and applied field angle. Different orientations may be better suited for different 
applications. Also, in a BHJ solar cell, the interface is a complex interpenetration between $\mathrm{D}$ and $\mathrm{A}$ molecules. This means that depending on the site in which the exciton will dissociate in the CT state, the field will be at a different angle with respect to the interface. This study can give an insight on the CT dissociation in such a system.

The inclusion of polarization in the model is shown to increase CT state splitting. In particular, this is due to the fact that the CT state studied is in the ground state, one electron on the LUMO of a $\mathrm{C}_{60}$ molecule and one hole on the HOMO of an anthracene molecule. When this is the case, the field needed to split the CT state in our simulations is very high $(\approx 0.5 \mathrm{~V} / \mathrm{cm})$. When one of the two charges composing the CT state moves, the Coulombic interactions binding the CT state decrease, and the field is so high that at least one of the two charges is in deep inverse Marcus region and cannot move to the electrode. This fact usually prevents the full splitting of the CT state. As discussed in sec. 6.2 and in Paper II, the effect of polarization and in particular of the external reorganization energy is to shift the Marcus region to much higher fields compared to the case in which polarization is neglected. This phenomenon has been shown to increase the probability of the complete CT state splitting (Paper III). 
[1] D. Mulvaney, V. Bolam, M. Cendejas, S. Davis, L. Ornelas, S. Kim, S. Mau, W. Rowan, E. Sanz, P. Satre, A. Sridhar, D. Young, and K. MacDonald. Toward a just and sustainable solar energy industry. a silicon valley toxics coalition white paper. Technical report, Silicon Valley Toxics Coalition (2009).

[2] S. R. Forrest. The path to ubiquitous and low-cost organic electronic appliances on plastic. Nature, 428, 911 (2004).

[3] J.-H. Kim, M. J. Han, and S. Seo. Flexible, stretchable, and patchable organic devices integrated on freestanding polymeric substrates. Journal of Polymer Science Part B: Polymer Physics, 53, 453 (2015).

[4] A. Facchetti. $\pi$-Conjugated Polymers for Organic Electronics and Photovoltaic Cell Applications. Chemistry of Materials, 23, 733 (2011).

[5] D. J. Gundlach. Low power, high impact. Nature Materials, 6, 173 (2007).

[6] D. Wohrle and M. Dieter. Organic Solar Cells. Advanced Materials, 3, 129 (1991).

[7] W. Shockley and H. J. Queisser. Detailed Balance Limit of Efficiency of p-n Junction Solar Cells. Journal of Applied Physics, 32, 510 (1961).

[8] M. A. Green. Third generation photovoltaics: Ultra-high conversion efficiency at low cost. Progress in Photovoltaics: Research and Applications, 9, 123 (2001).

[9] S. Xiao and S. Xu. High-Efficiency Silicon Solar Cells-Materials and Devices Physics. Critical Reviews in Solid State and Materials Sciences, 39, 277 (2014).

[10] F. H. Alharbi and S. Kais. Theoretical limits of photovoltaics efficiency and possible improvements by intuitive approaches learned from photosynthesis 
and quantum coherence. Renewable and Sustainable Energy Reviews, 43, 1073 (2015).

[11] M. A. Green, K. Emery, Y. Hishikawa, W. Warta, and E. D. Dunlop. Solar cell efficiency tables (Version 46). Progress in Photovoltaics: Research and Applications, 23, 805 (2015).

[12] M. Jørgensen, K. Norrman, F. C. Krebs, M. Jorgensen, K. Norrman, and F. C. Krebs. Stability/degradation of polymer solar cells. Solar Energy Materials and Solar Cells, 92, 686 (2008).

[13] S. Locci. Modeling of Physical and Electrical Characteristics of Organic Thin Film Transistors. Ph.D. thesis, University of Cagliari (2009).

[14] Y. V. Aulin. Excitons in Organic Semiconductors. Master's thesis, University of Groningen (2010).

[15] E. V. Hauff. The Role of Molecular Structure and Conformation in Polymer Electronics. Semiconductors and Semimetals, 85, 231 (2011).

[16] K. Vandewal and K. Tvingstedt. Charge Transfer States in Organic Donor-Acceptor Solar Cells. Semiconductors and Semimetals, 85, 261 (2011).

[17] C. Deibel. Photocurrent Generation in Organic Solar Cells. Semiconductors and Semimetals, 85, 297 (2011).

[18] M. A. Alam. Solar cells lecture 5: Organic photovoltaics. http://nanohub.org/resources/11950, Purdue Summer School (2011). Accessed: 2015-09-08.

[19] X. Zhu, Q. Yang, and M. Muntwiler. Charge-Transfer Excitons at Organic. Accounts of Chemical Research, 42, 1779 (2009).

[20] J. Lee, K. Vandewal, S. R. Yost, M. E. Bahlke, L. Goris, M. A. Baldo, J. V. Manca, and T. Van Voorhis. Charge transfer state versus hot exciton dissociation in polymer-fullerene blended solar cells. Journal of the American Chemical Society, 132, 11878 (2010).

[21] V. I. Arkhipov, P. Heremans, and H. Bässler. Why is exciton dissociation so efficient at the interface between a conjugated polymer and an electron acceptor? Applied Physics Letters, 82, 4605 (2003).

[22] A. V. Nenashev, M. Wiemer, F. Jansson, and S. D. Baranovskii. Theory to exciton dissociation at the interface between a conjugated polymer and an electron acceptor. Journal of Non-Crystalline Solids, 358, 2508 (2012).

[23] S. Verlaak, D. Beljonne, D. Cheyns, C. Rolin, M. Linares, F. Castet, J. Cornil, and P. Heremans. Electronic Structure and Geminate Pair Energetics at Organic-Organic Interfaces: The Case of Pentacene/ $\mathrm{C}_{60}$ Heterojunctions. Advanced Functional Materials, 19, 3809 (2009). 
[24] J. Cornil, S. Verlaak, N. Martinelli, A. Mityashin, Y. Olivier, T. Van Regemorter, G. D'Avino, L. Muccioli, C. Zannoni, F. Castet, D. Beljonne, and P. Heremans. Exploring the energy landscape of the charge transport levels in organic semiconductors at the molecular scale. Accounts of Chemical Research, 46, 434 (2013).

[25] R. R. Lunt, N. C. Giebink, A. Belak, J. B. Benziger, and S. R. Forrest. Exciton diffusion lengths of organic semiconductor thin films measured by spectrally resolved photoluminescence quenching. J. Appl. Phys., 105, 053711 (2009).

[26] G. A. Buxton and N. Clarke. Computer simulation of polymer solar cells. Modelling and Simulation in Materials Science and Engineering, 15, 13 (2007).

[27] F. Mirjani, N. Renaud, N. Gorczak, and F. C. Grozema. Theoretical Investigation of Singlet Fission in Molecular Dimers: The Role of Charge Transfer States and Quantum Interference. The journal of physical chemistry C, 118, 14192 (2014).

[28] Y. Tao, K. Yuan, T. Chen, P. Xu, H. Li, R. Chen, C. Zheng, L. Zhang, and W. Huang. Thermally Activated Delayed Fluorescence Materials Towards the Breakthrough of Organoelectronics. Advanced materials, pp. 1-28 (2014).

[29] K. Hannewald, V. Stojanović, J. Schellekens, P. Bobbert, G. Kresse, and J. Hafner. Theory of polaron bandwidth narrowing in organic molecular crystals. Physical Review B, 69, 075211 (2004).

[30] F. Ortmann, F. Bechstedt, and K. Hannewald. Theory of charge transport in organic crystals: Beyond Holstein's small-polaron model. Physical Review B, 79,235206 (2009).

[31] F. Ortmann, F. Bechstedt, and K. Hannewald. Charge transport in organic crystals: Theory and modelling. Physica Status Solidi (B), 248, 511 (2011).

[32] L. J. A. Koster, E. C. P. Smits, V. D. Mihailetchi, and P. W. M. Blom. Device model for the operation of polymer/fullerene bulk heterojunction solar cells. Physical Review B, 72, 085205 (2005).

[33] M. Gruber, B. Stickler, G. Trimmel, F. Schürrer, and K. Zojer. Impact of energy alignment and morphology on the efficiency in inorganic-organic hybrid solar cells. Organic Electronics, 11, 1999 (2010).

[34] C. de Falco, M. Porro, R. Sacco, and M. Verri. Multiscale modeling and simulation of organic solar cells. Computer Methods in Applied Mechanics and Engineering, 245-246, 102 (2012).

[35] H. Bässler. Charge Transport in Disordered Organic Photoconductors. A Monte Carlo Study. Physica Status Solidi (B), 175, 15 (1993).

[36] P. K. Watkins, A. B. Walker, and G. L. B. Verschoor. Dynamical Monte Carlo Modelling of Organic Solar Cells : The Dependence of Internal Quantum 
Efficiency on Morphology Dynamical Monte Carlo Modelling of Organic Solar Cells : The Dependence of Internal Quantum Efficiency on Morphology. Nano letters, 5, 1814 (2005).

[37] H. Van Eersel, R. a. J. Janssen, and M. Kemerink. Mechanism for efficient photoinduced charge separation at disordered organic heterointerfaces. Advanced Functional Materials, 22, 2700 (2012).

[38] V. Rühle, A. Lukyanov, F. May, M. Schrader, T. Vehoff, J. Kirkpatrick, B. Baumeier, and D. Andrienko. Microscopic Simulations of Charge Transport in Disordered Organic Semiconductors. Journal of Chemical Theory and Computation, 7, 3335 (2011).

[39] M. Jakobsson, M. Linares, and S. Stafström. Monte Carlo simulations of charge transport in organic systems with true off-diagonal disorder. The Journal of chemical physics, 137, 114901 (2012).

[40] M. J. Abraham, D. van der Spoel, E. Lindahl, B. Hess, and the GROMACS development team. GROMACS User Manual version 5.0.7. www.gromacs.org (2015).

[41] F. Jensen. Introduction to Computational Chemistry. Wiley (2007).

[42] W. L. Jorgensen and J. Tirado-Rives. The OPLS Potential Functions for Proteins. Energy Minimizations for Crystals of Cyclic Peptides and Crambin. Journal of the American Chemical Society, 110, 1657 (1988).

[43] W. L. Jorgensen, D. S. Maxwell, and J. Tirado-rives. Development and Testing of the OPLS All-Atom Force Field on Conformational Energetics and Properties of Organic Liquids. J. Am. Chem. Soc., 118, 11225 (1996).

[44] B. Hess, C. Kutzner, D. Van Der Spoel, and E. Lindahl. GROMACS 4: Algorithms for highly efficient, load-balanced, and scalable molecular simulation. Journal of Chemical Theory and Computation, 4, 435 (2008).

[45] D. Van Der Spoel, E. Lindahl, B. Hess, G. Groenhof, A. E. Mark, and H. J. C. Berendsen. GROMACS: Fast, flexible, and free. Journal of Computational Chemistry, 26, 1701 (2005).

[46] E. Lindhal, B. Hess, and D. Van Der Spoel. Gromacs 3.0: a package for molecular simulation and trajectory analysis. J . Mol. Model, 7, 306 (2001).

[47] H. Berendsen, D. van der Spoel, and R. van Drunen. GROMACS: A messagepassing parallel molecular dynamics implementation. Computer Physics Communications, 91, 43 (1995).

[48] V. Rühle. Berendsen and Nose-Hoover thermostats. http://www2.mpipmainz.mpg.de/ andrienk/journal_club/thermostats.pdf, University of Mainz (Journal Club) (2008). Accessed: 2015-09-08. 
[49] W. G. Hoover. Canonical dynamics: Equilibrium phase-space distributions. Physical Review A, 31, 1695 (1985).

[50] H. C. Andersen. Molecular dynamics simulations at constant pressure and/or temperature. The Journal of Chemical Physics, 72, 2384 (1980).

[51] S. Nosé. A molecular dynamics method for simulations in the canonical ensemble. Molecular Physics, 100, 191 (2002).

[52] M. Parrinello and A. Rahman. Polymorphic Transitions in Single Crystals: a New Molecular Dynamics Method. Journal of Applied Physics, 52, 7182 (1981).

[53] M. C. Heiber and A. Dhinojwala. Efficient Generation of Model Bulk Heterojunction Morphologies for Organic Photovoltaic Device Modeling. Physical Review Applied, 2, 014008 (2014).

[54] Ising model. http://en.wikipedia.org/wiki/Ising_model, Wikipedia: The free encyclopedia. Accessed: 2015-09-08.

[55] W. Cai. ME346A Introduction to Statistical Mechanics: Handout 12. Ising Model. http://micro.stanford.edu/ caiwei/me334/ME346A_Lecture_Notes_Win2012.pdf, Stanford University (2011). Accessed: 2015-10-20.

[56] J. A. Pople, D. P. Santry, and G. A. Segal. Approximate Self-Consistent Molecular Orbital Theory. I. Invariant Procedures. The Journal of Chemical Physics, 43, S129 (1965).

[57] J. A. Pople and G. A. Segal. Approximate Self-Consistent Molecular Orbital Theory. II. Calculations with Complete Neglect of Differential Overlap. The Journal of Chemical Physics, 43, S136 (1965).

[58] J. A. Pople. Approximate Self-Consistent Molecular-Orbital Theory. V. Intermediate Neglect of Differential Overlap. The Journal of Chemical Physics, 47, 2026 (1967).

[59] M. C. Zerner, G. H. Loew, R. F. Kirchner, and U. T. Mueller-Westerhoff. An intermediate neglect of differential overlap technique for spectroscopy of transition-metal complexes. Ferrocene. Journal of the American Chemical Society, 102, 589 (1980).

[60] H. P. and W. Kohn. Inhomogeneous electron gas. Physical Review, 136, 864 (1964).

[61] W. Kohn. Nobel Lecture: Electronic structure of matter-wave functions and density functionals. Rev. Mod. Phys., 71, 1253 (1999).

[62] W. Kohn and L. J. Sham. Self-Consistent Equations Including Exchange and Correlation Effects. Physical Review B, 140 (1965). 
[63] A. Melianas, F. Etzold, T. J. Savenije, F. Laquai, O. Inganäs, and M. Kemerink. Photo-generated carriers lose energy during extraction from polymer-fullerene solar cells. Nature Communications, 6, 8778 (2015).

[64] R. Marcus. On the Theory of Oxidation-Reduction Reactions Involving Electron Transfer. I. The Journal of Chemical Physics, 24, 966 (1956).

[65] L. V. and R. Dogonadze. Theory of non-radiation electron transitions from ion to ion in solutions. Dok. Akad. Nauk SSSR, 124, 123 (1959).

[66] L. V. Present state of the theory of oxidation-reduction in solution (bulk and electrode reactions). Adv. Electrochem. Electrochem. Eng., 4, 249 (1966).

[67] J. J. Temperature dependent activation energy for electron transfer between biological molecules. J. Chem. Phys., 64, 4860 (1976).

[68] K. Asadi, A. J. Kronemeijer, T. Cramer, L. Jan Anton Koster, P. W. M. Blom, and D. M. de Leeuw. Polaron hopping mediated by nuclear tunnelling in semiconducting polymers at high carrier density. Nature Communications, 4, 1710 (2013).

[69] R. Pratesi. Struttura della materia (lecture notes), Florence University (2007/2008).

[70] M. Hoffmann. Mixing of Frenkel and Charge-Transfer Excitons and Their Quantum Confinement in Thin Films. Elsevier (2003).

[71] F. Essler, H. Frahm, F. Göhmann, A. Klümper, and V. Korepin. The OneDimensional Hubbard Model. Cambridge University Press (2005). Chapter 1 .

[72] T. Holstein. Studies of polaron motion : Part I. Annals of Physics, 8, 325 (1959).

[73] T. Holstein. Studies of polaron motion : Part II. Annals of Physics, 8, 343 (1959).

[74] Monte Carlo method. http://en.wikipedia.org/wiki/Monte_Carlo_method, Wikipedia: The free encyclopedia. Accessed: 2015-09-08.

[75] N. Metropolis. The Beginning of the Monte Carlo Method. Los Alamos Science, 15, 125 (1987).

[76] N. Metropolis and S. Ulam. The monte carlo method. Journal of the American Statistical Association, 44, 335 (1949).

[77] C. Andrieu, A. Doucet, and R. Holenstein. Particle Markov chain Monte Carlo methods. Journal of the Royal Statistical Society: Series B (Statistical Methodology), 72, 269 (2010). 
[78] E. F. Valeev, V. Coropceanu, D. A. da Silva Filho, S. Salman, and J.-L. Brédas. Effect of Electronic Polarization on Charge-Transport Parameters in Molecular Organic Semiconductors. J. Am. Chem. Soc., 128, 9882 (2006).

[79] F. Castet, P. Aurel, A. Fritsch, L. Ducasse, D. Liotard, M. Linares, J. Cornil, and D. Beljonne. Electronic polarization effects on charge carriers in anthracene: A valence bond study. Physical Review B, 77, 115210 (2008).

[80] J. Cornil, D. Beljonne, J.-P. Calbert, and J.-L. Brédas. Advanced materials, 13, 1053-1067 (2001).

[81] R. Mulliken, C. Rieke, D. Orloff, and H. Orloff. Formulas and Numerical Tables for Overlap Integrals. Journal of Chemical Physics, 17, 1248 (1949).

[82] A. Hansson and S. Stafström. Intershell conductance in multiwall carbon nanotubes. Physical Review B, 67, 075406 (2003).

[83] J. Kirkpatrick. An Approximate Method for Calculating Transfer Integrals Based on the ZINDO Hamiltonian. International Journal of Quantum Chemistry, 108, 51 (2008).

[84] B. Baumeier, J. Kirkpatrick, and D. Andrienko. Density-functional based determination of intermolecular charge transfer properties for large-scale morphologies. Physical chemistry chemical physics : PCCP, 12, 11103 (2010).

[85] J.-L. Brédas, D. Beljonne, V. Coropceanu, and J. Cornil. Charge-transfer and energy-transfer processes in pi-conjugated oligomers and polymers: a molecular picture. Chemical reviews, 104, 4971 (2004).

[86] A. Miller and E. Abrahams. Impurity Conduction at Low Concentrations. Phys. Rev., 120, 745 (1960).

[87] D. H. Dunlap, V. M. Kenkre, and P. E. Parris. What is behind the $\sqrt{E}$ ? Journal of Imaging Science and Technology, 43, 437 (1999).

[88] S. Novikov and V. A. Distribution of electrostatic potential in a lattice of randomly oriented dipoles. Journal of Experimental and Theoretical Physics, 79, 482 (1994).

[89] S. Novikov and V. A. Cluster Structure in the Distribution of the Electrostatic Potential in a Lattice of Randomly Oriented Dipoles. Journal of Physical Chemistry, 99, 14573 (1995).

[90] J. Frenkel. On Pre-Breakdown Phenomena in Insulators and Electronic SemiConductors. Physical Review, 54, 647 (1938).

[91] I. I. Fishchuk, A. Kadashchuk, S. V. Novikov, M. Ullah, J. Genoe, N. S. Sariciftci, H. Sitter, and H. Bässler. Origin of electric field dependence of the charge mobility and spatial energy correlations in c60-based field effect transistors. Molecular Crystals and Liquid Crystals, 589, 18 (2014). 
[92] A. Pivrikas, M. Ullah, C. Simbrunner, H. Sitter, H. Neugebauer, and N. Serdar Sariciftci. Comparative study of bulk and interface transport in disordered fullerene films. Physica Status Solidi (B) Basic Research, 248, 2656 (2011).

[93] E. N. Parker. Tensor Virial Equations. Physical Review, 96, 1686 (1954). 


\section{List of included Publications}

[I] Transition fields in organic materials: From percolation to inverted Marcus regime. A consistent Monte Carlo simulation in disordered PPV

R. Volpi, S. Stafström, M. Linares

Journal of Chemical Physics 142, 094503 (2015)

[II] Effect of Polarization on the Mobility of $\mathrm{C}_{60}$ : A Kinetic Monte-Carlo Study

R. Volpi, S. Kottravel, M. Pedersen, S. Stafström, M. Linares

Submitted

[III] Charge-transfer state dynamics at $\mathbf{C}_{60}$-anthracene interfaces: a kinetic Monte Carlo approach

R. Volpi, R. Nassau, M. Linares

Manuscript 


\section{Contribution to the Papers}

\section{Paper I}

I have been introduced to the project by my supervisor Mathieu Linares and my co-supervisor Sven Stafström and I played an active role in the conception of the theoretical model. I wrote the code based on a previously existing code of the project. I executed and analyzed all the calculations, and I wrote the manuscript.

\section{Paper II}

I proposed the polarization part of the theoretical model used during discussion with my supervisor Mathieu Linares and I wrote the code. With the help of Mathieu Linares and my co-supervisor Sven Stafström I planned executed and analyzed all the calculations, and I wrote the manuscript.

\section{Paper III}

I wrote the code allowing the treatment of multiple charges and I was involved in the supervision of the master student Racine Nassau. With my help and the help of Mathieu Linares, Racine planned and executed all the calculations. I helped her with the analysis of the data and I participated to the writing of the manuscript. 


\section{Papers}

The articles associated with this thesis have been removed for copyright reasons. For more details about these see:

http://urn.kb.se/resolve?urn=urn:nbn:se:liu:diva-122991 